The effects of psychological distance on abstraction: Two meta-analyses

Courtney K. Soderberg ${ }^{1}$

Shannon P. Callahan ${ }^{2}$

Annie O. Kochersberger ${ }^{2}$

Elinor $\mathrm{Amit}^{3}$

Alison Ledgerwood ${ }^{2}$

${ }^{1}$ Center for Open Science

${ }^{2}$ University of California, Davis

${ }^{3}$ Harvard University

Keywords: Construal level, psychological distance, temporal distance, mental

representation, meta-analysis

Contact information:

Courtney K. Soderberg

Center for Open Science

210 Ridge McIntire Road, Suite 500

Charlottesville, VA 22903

Email: csoderbe@gmail.com 


\begin{abstract}
Psychological distance and abstraction both represent key variables of considerable interest to researchers across cognitive, social, and developmental psychology. Moreover, largely inspired by construal level theory, numerous experiments across multiple fields have now connected these two constructs, examining how psychological distance affects the level of abstraction at which people mentally represent the world around them. The time is clearly ripe for a quantitative synthesis to shed light on the relation between these constructs and investigate potential moderators. To this end, we conducted two metaanalyses of research examining the effects of psychological distance on abstraction and its downstream consequences. Across 106 papers containing a total of 267 experiments, our results showed a reliable and medium-sized effect of psychological distance on both level of abstraction in mental representation and the downstream consequences of abstraction. Importantly, these effects replicate across time, researchers, and settings. Our analyses also identified several key moderators, including the size of the difference in distance between two levels of a temporal distance manipulation and the dependent variable's capacity to tap processing of both abstract and concrete features (rather than only one or the other). We discuss theoretical and methodological implications, and highlight promising avenues for future research.
\end{abstract}




\section{The effects of psychological distance on abstraction: Two meta-analyses}

People spend much of their lives immersed in their direct experience, focusing on the self, here, and now. Yet humans also possess a potentially unique capacity for mental travel - they can remember the past and plan for the future, coordinate action at a distance, relate to other people, and imagine hypothetical alternatives to reality. What psychological processes enable people to transcend their immediate experience in such ways? This key question lies at the heart of considerable research on memory, prospection, perspective-taking, communication, and learning, and continues to occupy a central place in theory and research across multiple domains (e.g., Clark \& Brennan, 1991; Leslie, 1987; Mischel, Shoda, \& Rodriguez, 1989; Schacter, 2012; Suddendorf \& Corballis, 2007).

Likewise, research across numerous disciplines has sought to understand the antecedents and consequences of mental abstraction, or the process of constructing a mental representation that focuses on the essential characteristics of an object while omitting less relevant details. Abstraction is thought to play a fundamental role in a number of basic psychological processes, including categorization, language development, communication, causal inference, and self-control, and is therefore the subject of considerable empirical attention in disciplines ranging from cognitive and clinical psychology to neuroscience, linguistics, and marketing (e.g., Badre \& d'Esposito, 2007; Rosch et al., 1976; Semin \& Fiedler 1988; see Burgoon, Henderson, \& Markman, 2013, for a review).

The notion that mental abstraction might provide a key mechanism by which humans can move beyond direct experience was first proposed within the framework of 
construal level theory (CLT), which posits that psychological distance systematically influences the way that people mentally represent the world around them (Liberman \& Trope, 2008; Trope \& Liberman, 2010). Moreover, by changing the level at which an object or event is subjectively construed, psychological distance can also have consequential implications for a wide range of important outcomes, including voting intentions, stereotyping, prosocial behavior, self-control, and creativity (Trope \& Liberman, 2010).

Perhaps because it connects two constructs that have been central to so many research domains, the notion that psychological distance might affect mental representation has precipitated a considerable amount of empirical work. A quick Google Scholar search reveals that the initial empirical paper proposing such a link (Liberman \& Trope, 1998) has been cited over 700 times, and the first conceptual paper on construal level theory (Trope \& Liberman, 2003) has been cited over 1200 times. Our literature search for studies linking these two concepts, detailed below, revealed hundreds of empirical articles published with increasing frequency in fields ranging from social and cognitive psychology to consumer behavior to technology.

In light of the abundance of individual studies that have explored the relation between psychological distance and construal level, it is perhaps surprising that researchers have yet to quantitatively synthesize this rapidly expanding area of research using the tool of meta-analysis. Given the critical importance of meta-analyses in advancing knowledge and refining theory (Chan \& Arvey, 2012; Hunter \& Schmidt, 2004), the time seems ripe for such an undertaking. Moreover, meta-analysis could afford new theoretical insights by providing an opportunity to test several potential moderators. 
In this paper, we briefly review the theoretical rationale for expecting a link between psychological distance and abstraction as well as a few illustrative examples of typical experiments testing this link. Next, we provide an overview of two meta-analyses that examined the effect of distance on (1) construal level and (2) the downstream consequences of abstraction for evaluation, prediction, and behavior. We detail our specific moderator variables, predictions, and coding in the methods section, and then describe the results and their implications for theory and future research.

\section{Distance and Abstraction}

The idea that distance and abstraction might be related begins with the question of how people expand and contract their mental horizons - that is, how individuals are able to think about times, places, people, and possibilities that vary in their distance from directly experienced "me, here, and now." To address this question, construal level theory builds on the basic idea that the same object or event can be mentally represented (or construed) at varying levels of abstraction (Medin, 1989; Rosch, Mervis, Gray, Johnson, \& Boyes-Braem, 1976; Trope \& Liberman, 2003; Vallacher \& Wegner, 1987). In other words, mental representations can be arranged along a vertical continuum of abstraction, from low to high. Higher-level construal involves constructing mental representations that are relatively abstract and structured, extracting the central, superordinate, and goalrelevant features of an object or event and leaving out specific and peripheral details. In contrast, lower-level construal involves constructing more concrete representations that include an object's detailed, subordinate, and context-specific features. Such representations tend to lose the structure that separates important from peripheral and irrelevant aspects. For example, construing a piece of pie at a lower level of abstraction 
might involve representing it as a homemade blueberry pie with a lattice crust, whereas construing the same piece of pie at a higher level of abstraction might involve representing it as a dessert (see Table 1 for illustrative examples of how level of construal has been operationalized in past research).

According to construal level theory, individuals can mentally traverse psychological distance by forming increasingly abstract mental representations of objects and events. Because abstraction enables people to cross the distances that separate self from other, now from then, and here from there in this way, the psychological distance of an object or event should systematically influence the level at which people mentally represent it. Psychological distance refers to any dimension along which an object or event can be removed from the self, here, now, and reality. The theory identifies four key dimensions of psychological distance - temporal, spatial, social, and likelihood or hypotheticality — and posits that because they share a common egocentric reference point, they should all similarly influence level of construal.

The link between distance and abstraction is thought to reflect a functional advantage to using higher-level construal when thinking about more psychological distant objects or events, because higher-level representations are less likely to change across contexts. For example, asking someone to "play a CD" is only effective in times and places where CDs exist, and when communicating with people who have them. In contrast, the request to "play some music" retains its meaning across many more times, places, and individuals. The more abstract representation of "playing music" can encompass a broad range of specific and concrete instantiations (e.g., a CD, an mp3, or strumming a guitar) and therefore travels better across different distances. 
The effect of psychological distance on construal level was first studied in terms of temporal distance (Liberman \& Trope, 1998). In one study, participants were asked to think of themselves performing activities either "tomorrow" or "next year" and were then given the Behavioral Identification Form (BIF; Vallacher \& Wegner, 1989), a measure that assesses the extent to which people prefer to describe actions (e.g., "locking a door") in terms of low-level, specific means (e.g., "putting a key in the lock") or high-level, superordinate goals (e.g., "securing the house"). Participants showed a greater relative preference for the high-level versus low-level descriptions when they imagined performing the activities in the more distant (vs. near) future, consistent with the notion that people construe events more abstractly as temporal distance increases.

Later research extended the theory to spatial distance (e.g., Fujita, Henderson, Eng, Trope, \& Liberman, 2006), social distance (e.g., Liviatan, Trope, \& Liberman, 2008), and hypotheticality or likelihood (e.g., Wakslak, Trope, Liberman, \& Alony, 2006; example manipulations for the four types of distance can be found in Table 2). For instance, Fujita et al. (2006) asked New York University (NYU) students to watch a video that ostensibly had been filmed at either NYU's Manhattan campus (spatially near condition) or NYU's study abroad campus in Florence, Italy (spatially distant condition) and then asked them to write about what they saw. Participants' open-ended responses were coded for abstractness of language using a coding scheme based on the Linguistic Category Model (Semin \& Fielder, 1988). Consistent with the notion that people use more abstract representations to think about more psychologically distant objects and events, participants used more abstract language to describe the video when they thought it had been filmed in Florence rather than Manhattan. 
Researchers have found comparable effects across various operationalizations of psychological distance and construal level, including perceptual as well as conceptual measures of construal. For instance, Wakslak, Trope, Liberman, and Alony (2006) manipulated hypotheticality by leading participants to believe that there was a near certainty (a 95\% chance) or a more distant possibility (a 5\% chance) that they would be asked to complete the Gestalt Completion Test (GCT; Ekstrom, French, Harmon, \& Dermen, 1976) later in the experiment. The GCT is a perceptual measure of abstraction that presents participants with a series of fragmented pictures that they must try to identify; success on the task requires abstracting the gist of the object from the fragmented pieces to close the Gestalt. All participants were given several "practice items" from the GCT, which provided the dependent measure of perceptual construal. Participants in the distant (vs. near) condition — who believed they were less likely to complete the full GCT later on-showed greater abstraction on the practice problems.

\section{Downstream Consequences of Abstraction}

Because of the effect of psychological distance on level of mental representation, CLT also postulates that psychological distance can have a host of downstream consequences for prediction, evaluation, and behavior. In other words, distance changes the extent to which a person's mental representation of an object includes or weighs highand low-level features of the object, which in turn can affect the person's subsequent judgments and actions. For example, the desirability of a given option can be considered a primary, high-level feature of that option, whereas its feasibility can be considered a secondary, low-level feature (Liberman \& Trope, 1998). As a person's mental representations of an option become more abstract, desirability (vs. feasibility) 
considerations should be given greater weight, which in turn should affect people's preferences. Thus, the desirability (vs. feasibility) of an option should more strongly influence evaluations and choices when the option is more psychologically distant.

In research designed to test this prediction in the context of hypothetical distance, or likelihood, Todorov, Goren, and Trope (2007) asked participants to rate how willing they would be to sign up for several different promotional campaigns, some of which were very likely to occur and others that were very unlikely to occur. The campaigns also varied in whether their content was especially desirable to participants or especially feasible for them. As predicted, participants' willingness to sign up for the desirable options increased and their willingness to sign up for the feasible options decreased as the promotions became more unlikely (i.e., more distant from reality), presumably due to the effect of distance on the extent to which participants' mental representations of the options prioritized desirability versus feasibility concerns.

Similar effects on downstream consequences have been found with manipulations that involve varying temporal, spatial, and social distance. For example, Liviatan et al. (2008) asked participants to evaluate short stories written by socially near or distant others. They manipulated social distance by varying how similar the writer was to each participant: In the socially near condition, the writer was taking the same courses as the participant, whereas in the socially distant condition, the writer took different courses. The stories also varied in their writing quality (a primary, important characteristic for evaluating the quality of the short story) and in the ostensible ability of the writer in an irrelevant domain (a secondary, unimportant characteristic for evaluating the quality of the story). As hypothesized, distance increased the relative impact of the primary (vs. 
secondary) information on story evaluations. Whereas writing quality had a greater impact on participants' evaluations of stories written by socially distant (vs. close) others, the writer's ability in an irrelevant domain had a greater impact on participants' evaluations of stories written by socially close (vs. distant) others.

Taken together, then, numerous studies seem to suggest that variations in temporal, spatial, social, and hypothetical distance can influence the level of abstraction in mental representation. Moreover, these four distance dimensions appear to have a range of downstream consequences for evaluations, predictions, and behaviors in important domains ranging from negotiation and political behavior to health and cyber security (e.g. Choi, Park, \& Oh, 2011; Giacomantonia, De Dreu, \& Manetti, 2010; Menegatti \& Rubini, 2012; Tam, Glassman, \& Vandenwauver, 2010). Given the farreaching implications of the potential link between psychological distance and abstraction, as well as its relevance for researchers interested in studying these central constructs across different areas of psychological science, a quantitative synthesis of existing evidence seems both important and overdue.

\section{The Present Meta-Analysis}

In light of the large number of individual studies testing the effects of psychological distance on abstraction and its downstream consequences, conducted across a wide array of disciplines and using a wide variety of methodologies, metaanalysis provides an invaluable tool for synthesizing the existing knowledge in the literature and pointing the way toward important future directions. We had four objectives in conducting the meta-analyses described here. First, we wanted to estimate the magnitude of the effect of psychological distance on construal level and on the 
downstream consequences of abstraction. Especially given current concerns both within and beyond the field about diminishing effect sizes and failures to replicate (Ioannidis, 2005; Lehrer, 2010; Yong, 2012), meta-analysis provides a key method for estimating an effect with maximal accuracy and precision. Moreover, because a meta-analysis can provide a better estimate of this effect size than any one study, it can more accurately inform power analyses for future studies. Because psychology studies are often underpowered (Bakker, van Dijk, \& Wicherts, 2012), initial estimates of effect sizes are often inflated (Button et al., 2013); when power analyses for future studies use these inflated effect size estimates, they are also likely to be underpowered and thus less likely to replicate an effect even if the effect is real (Button et al., 2013; Perugini, Gallucci, \& Constantini, 2014). Meta-analyses can help address this problem.

Our second objective was to determine how robust the effects of psychological distance are across different researchers, settings, and samples. For instance, we tested whether researchers with direct ties to the original authors of the theory obtained similar effect sizes as independent laboratories. By exploring whether theory-irrelevant variations in researchers and settings moderate the size of the effect, we can examine its replicability and generalizability across a variety of different conditions.

Third, we were interested in testing some of the possibilities implied by construal level theory about the interchangeability of certain effects. For example, CLT distinguishes between temporal, spatial, social, and hypothetical distance but predicts that they should have similar effects on abstraction, as well as on evaluations, predictions, and behaviors (Trope \& Liberman, 2010, p. 440). Although a large number of individual studies suggest that all four types of distance can affect construal level, no studies have 
compared the magnitude of these effects to determine whether the four types of distance are equal in impact. Because a meta-analysis aggregates across many individual studies, it provides a prime tool for comparing the effect sizes resulting from manipulations of the four types of distance to determine whether they are equal or whether one type of distance may produce stronger effects than others (see Trope \& Liberman, 2010, p. 444, for a discussion of this possibility).

Finally, we wanted to look for potential moderators that might help provide new insights into the link between distance and abstraction. For example, CLT posits that distance will lead people to construct relatively more abstract mental representations, but it does not specify exactly how distance separately influences the processing of abstract and concrete information. It is possible that increased distance might only increase the processing of abstract information, only decrease the processing of concrete information, or both; all three possibilities could produce higher-level mental representations. Construal level theory is agnostic on this point: It simply makes a relative prediction about the extent to which mental representations will reflect abstract versus concrete information. Yet the question is both interesting and important if we are to fully understand the effects of psychological distance on mental representation.

Intriguingly, individual empirical studies do not always find symmetric effects of distance on the weighting or inclusion of high- and low-level features (e.g., Fujita, Eyal, Chaiken, Trope, \& Liberman, 2008; Ledgerwood, Wakslak, \& Wang, 2010), which could reflect a simple lack of power or a meaningful asymmetry. Meanwhile, some researchers have theorized that a meaningful asymmetry should exist, suggesting that abstract features are processed at both near and far distance points and that only the processing of 
concrete features changes across distance (Kim, Park, \& Wyer, 2009). By examining the effect sizes associated with dependent variables that measure changes in the processing of only abstract information, only concrete information, or a relative change in both, we will be able to shed new light on the process through which distance alters mental representations.

In order to accomplish these four goals, we investigated the average effect size of distance on construal level and the downstream consequences of abstraction, as well as a number of moderators related to the characteristics of the articles, the study samples, the distance manipulations and the dependent variables (enumerated below and listed in Table 3).

\section{Methods}

\section{Literature Search}

We used four procedures to obtain published and unpublished studies. First, we conducted an extensive search of computerized databases (PsycINFO, Web of Science, and PubMed) during August 2012 and again on November 5, 2013. The keywords used in the searches were words related to psychological distance and abstraction or to commonly used measures of construal level, including psychological distance, distance, construal level, abstraction, concrete, BIF, Gestalt Completion Test, and Navon task (see Appendix A for a full list of search strings). Second, we searched the reference sections of recent literature reviews and chapters for additional citations. Third, we sent multiple emails requesting relevant unpublished or in press studies to the Society for Personality and Social Psychology (SPSP) listserv and the Organizational Behavior Division listserv hosted by the Academy of Management, as well as directly to researchers who frequently 
conduct studies on psychological distance and construal level. Finally, we approached authors with relevant posters or symposia presentations at the 2013 SPSP conference in New Orleans to ask for information about their studies.

\section{Inclusion and Exclusion Criteria}

We used the following five criteria to determine whether each study was eligible for inclusion in the meta-analysis:

1. The study had to include a manipulation of one of the four dimensions of psychological distance (temporal, spatial, social, or hypothetical) and a measure that assessed either construal level or a downstream consequence of abstraction. Given that we were specifically interested in the causal impact of psychological distance on construal level, we did not include studies that did not manipulate distance (e.g., studies that only measured participants' subjective perception of distance or that measured personality traits, such as temporal orientation, that might be correlated with abstraction). In addition, we followed past theoretical work in distinguishing between the constructs of psychological distance (i.e., distance from directly-experienced me, here, now, and reality) and distancing or “distance-related" variables (such as novelty and familiarity, which can create a subjective sense of distance but are not themselves a dimension of distance from direct experience; see e.g., Trope \& Liberman, 2010). Because our meta-analysis aimed to examine the effects of distance, we did not include studies that manipulated these kinds of distancing variables rather than distance itself. ${ }^{1}$

\footnotetext{
${ }^{1}$ Essentially, this criterion meant that we only included studies that manipulated the extent to which an object was removed from a participant's direct experience along one of the four distance dimensions. In a handful of cases, the study manipulation fell close to this line (for instance, a study that manipulated whether people think about themselves from a first- vs. third-person perspective, or whether they view
} 
2. The levels of the manipulated distance variable had to differ in their absolute distance from direct experience. For example, a study in which an event was scheduled to take place either one week or one year from today would be included, as there would be an actual difference in time of 358 days between the two conditions. However, a study in which an event was described either one year in the past or one year in the future would not be included, as both conditions are the same objective distance (365 days) from direct experience.

3. The study could not include more than one manipulation intended to influence abstraction (e.g., a second manipulation of distance, a procedural priming manipulation intended to influence construal level, or a manipulation of a distancing variable). In this first meta-analysis of construal level research, we wanted to accurately estimate the effect of psychological distance on construal level. Including studies that simultaneously manipulated abstraction in multiple ways could artificially inflate or deflate the estimate of this effect. For example, if a study crossed a temporal distance manipulation with a spatial distance manipulation, the results would reflect what happens when distances match or mismatch, rather than how a single dimension of distance influences construal level. The question of how different conflicting distances or conflicting distance and construal level manipulations combine to affect abstraction, though outside the scope of this paper, is clearly an interesting question for future work.

images of their current location from a nearby or distant perspective, would be classified as "distancing" rather than "distance" because the object under consideration in both conditions was some aspect of me, here, or now - that is, the manipulation changed whether people considered the egocentric origin point from a more or less distanced perspective, rather than changing the distance between the object and the egocentric origin point). In these cases, the first and last author always discussed the inclusion decision to ensure it was consistent across studies. 
4. When a study tested boundary conditions for the effect (e.g., a condition in which people have no access to either low- or high-level information about an object), we did not include conditions for which the authors predicted that distance should not affect the dependent variable (since again, including conditions designed to block the effect of distance on abstraction would artificially influence our effect size estimate).

5. For feasibility reasons, the study had to be published in the language spoken by the authors (English).

After applying the above criteria to the studies compiled during the literature search, we obtained a sample of 267 studies from 106 articles for the meta-analyses. ${ }^{2}$

\section{Coding Procedures}

The authors jointly developed a coding manual that specified the coding categories and possible codes to be used for each study. Table 3 lists the moderators that were coded and a description of how each moderator was operationalized. Eligible studies were coded by the first three authors using the coding manual and a corresponding data coding spreadsheet. Given the large number of studies included in the meta-analysis, it was not feasible for two authors to code each variable for every study; we therefore randomly selected 50 studies to be coded by all three coders in order to obtain a measure of interrater reliability. The percent agreement between all three coders was high, $91 \%$. Because of the importance of the coding related to effect sizes, all dependent variable and effect size coding was performed by two independent coders, and all disagreements were resolved through discussion among the coders.

\footnotetext{
${ }^{2}$ Of the 164 articles that met our first inclusion criterion, 8 failed to meet criterion 2,27 failed to meet criterion 3, 6 failed criterion 5, and 17 did not provide enough information (in the article itself or after contacting the authors) to accurately calculate an effect size.
} 
Article characteristics. We coded for three variables related to the characteristics of the article or authors to explore the consistency of the effect size across different researchers and studies. To examine consistency across research laboratories, we coded for whether any of the authors of the paper trained under one of the two original authors of construal level theory (Nira Liberman or Yaacov Trope). To explore whether the effect might differ depending on whether it was conducted in the theory's "home discipline" of psychology, we coded for whether or not the study was published in a psychology journal. Finally, we coded for publication year to assess whether the effect sizes associated with construal level theory have decreased over time, a pattern that has been identified as a symptom of publication bias (Button et al., 2013; Schooler, 2011).

Study sample demographics. We coded for three variables related to the demographics of the participants in each study to determine the consistency of the effect across different populations. First, although construal level theory makes no predictions about gender, we coded the percentage of participants in the study sample who were female to explore the possibility of gender effects. Second, to explore culture as a potential moderator, we recorded the national individualism/collectivism score of the country in which the data was collected (Hofstede, Hofstede, \& Minkov, 2010). The general holistic worldview associated with more collectivistic cultures (Nisbett, Peng, Choi, \& Norenzayan, 2001; see also Kuhnen \& Oyserman, 2002) might suggest that people from these cultures tend to think more abstractly than those from individualistic cultures, which could perhaps limit or enhance the effect of psychological distance on abstraction. Finally, we coded for whether the study was conducted in a laboratory setting, a field setting (e.g., a classroom or mall), or online, to investigate the extent to 
which the effect generalized from carefully controlled laboratory environments to other types of settings.

Distance manipulation characteristics. We coded for four variables related to the distance manipulation used in each study to assess the extent to which methodological differences between studies might alter effect sizes. First, we coded for whether the distance described in the manipulation was portrayed to the participant as real (e.g., selecting an actual class to take either next semester vs. next year) or imagined (e.g., imagining the selection of a hypothetical class that could be taken next semester or next year). Researchers may intuitively assume that manipulations tend to be less powerful when described to participants as imaginary rather than real, and may therefore try to portray distance manipulations as real whenever possible; we wanted to explore whether this intuition is valid. Second, we coded for the type of distance manipulated in the study (temporal, spatial, social, or hypothetical). As previously mentioned, construal level theory treats these distances as interchangeable and predicts that they should have similar (though not necessarily equal) effects on abstraction. A meta-analysis is ideally suited to test this prediction.

Finally, for studies that manipulated temporal distance, we coded for whether the distance was in the past or future, the number of days from today for the time point specified in the near and distant conditions, and the difference in days between the near and distant conditions. For instance, if a study manipulated temporal distance by changing whether a policy would be implemented next month or next year, we coded the near-future condition as 30 days, the distant-future condition as 365 days, and the difference between the two conditions as 335 days. We did this in order to explore the 
specific nature of the relation between distance and construal level (e.g., linear, step-wise, or curvilinear), which we discuss in more detail later.

Dependent variable characteristics. For direct measures of construal level, we coded whether the measure was perceptual (e.g., the Gestalt Completion Task; Ekstrom et al., 1976) or conceptual (e.g., the BIF; Vallacher \& Wegner, 1989; see Trope \& Liberman (2010) for more on this distinction). We also coded for whether the dependent variable measured processing of abstract information, concrete information, or the relative difference in the processing of the two types of information (see Table 4 for example dependent variables in each category). As previously mentioned, increasing psychological distance could increase the processing of high-level features, decrease the processing of low-level features, or both. All three are consistent with the theory, but we sought to more accurately specify the process by which distance influenced mental representations in our meta-analysis.

For measures that captured downstream consequences of abstraction, we coded for whether the downstream dependent variable measured an evaluation (e.g., favorability toward a political policy), prediction (e.g., predicting what others will do in the future based on either an overall past trend or a recent deviation from that trend), or behavior (e.g., the extent to which people cooperate during negotiations), to explore whether distance similarly affected these three types of outcomes (see Table 5 for additional examples of measures assessing downstream consequences of construal level).

\section{Computation of Effect sizes}

Only studies that met all inclusion criterion and that contained adequate information to accurately calculate an effect size were included in the analyses. If a 
publication did not contain sufficient information to compute an effect size, we emailed the corresponding author to ask for the necessary information; if the author did not reply or could not provide the necessary information, the potential effect size could not be included.

One hundred and six articles contained at least one study that met our inclusion criterion. We distinguished between dependent variables that assessed the effect of psychological distance on construal level and those that measured the effect of psychological distance on a downstream consequence of construal level (i.e., evaluations, predictions, and behaviors), which resulted in two groups of effects. In total, our sample included 289 effect sizes from 118 studies with direct measures of construal level, and 428 effect sizes from 182 studies that measured downstream consequences of abstraction. (Note that a number of studies contained both direct and downstream consequences measures, and so were allowed to contribute effect sizes to both groups.) We conducted two separate meta-analyses on the two groups of studies using the same analytic procedures.

Because meta-analytic procedures assume that all included effect sizes measure the same effect (Card, 2012; Cooper, Hedges, \& Valentine, 2009), we needed to ensure that the effect sizes for all studies were calculated in a comparable way. We therefore calculated all effect sizes based on the difference between the near and distant conditions, with a positive effect size indicating greater abstraction in the distant versus near condition (i.e., a theory-consistent effect of distance on abstraction). For studies with crossed designs, we calculated effect sizes that reflected the impact of distance at each level of the moderator. This ensured that the effect sizes from studies with different 
designs were comparable, so that we could include both simple two-cell designs and more complex interaction designs in the same meta-analysis.

Given that all studies in our sample contained a dichotomous manipulation of psychological distance and that the majority included a continuous dependent measure, we used Hedges' $g$ as our measure of effect size. Hedges' $g$ is a bias-corrected Cohen's d that corrects for the fact that in small sample studies, Cohen's d tends to overestimate the true effect size (Card, 2012; Borenstein, 2009). All effect sizes were transformed into a Hedges' g effect size, calculated using the Comprehensive Meta-analysis 2 software (Borenstein, Hedges, Higgins, \& Rothstein, 2005).

\section{Meta-analytic Procedures}

Combination of multiple effect sizes within studies. The majority of studies in our sample could contribute more than one effect size to our meta-analyses because they included multiple dependent variables and/or multiple pairwise comparisons (as in the case of studies with a $2 \times 2$ design). However, multiple effect sizes from a single study are statistically dependent, and so including them poses a problem for assumptions of statistical independence in the meta-analytic calculations (Card, 2012; Cooper et al., 2009; Cooper, 2009).

We followed the typical strategy for dealing with statistically dependent effect sizes, the shifting unit of analysis (Card, 2012; Cooper, 2009), which involves using the study as the unit of analysis for the estimate of the overall average effect size. ${ }^{3}$ Thus, each study is allowed to contribute only one effect size, calculated as a weighted average of

\footnotetext{
${ }^{3}$ A different approach is to use the correlated effect size method; however, this strategy requires an average of at least five effect sizes per study (which we did not have), and a recently-proposed small sample correction requires more degrees of freedom for the moderators than we had in our sample for the parameter estimates to be stable (see Hedges, Tipton, \& Johnson, 2010; Tanner-Smith \& Tipton, in press; Tipton, in press).
} 
the effect sizes in each study. Following this approach, we computed all pertinent effect sizes for each study, and then calculated one weighted average of all the direct effects for the first meta-analysis, and another weighted average off all the downstream effects for the second meta-analysis. These weighted averages were then used as the unit of analysis for computing the overall effect sizes in both meta-analyses.

Analyses that tested potential moderating variables proceeded in one of two ways. If the value of the moderator was consistent across all of the effect sizes in a study (e.g., year of publication), then we used the average effect sizes as described above. However, when the value of a moderator was inconsistent across different effect sizes in a study, it was allowed to contribute one effect size to each level of the moderator (see Cooper, 2009). For example, if a study contained both a perceptual and a conceptual dependent variable, those effect sizes would not be averaged together during the analysis investigating whether perceptual and conceptual measures have equal effect sizes. However, for the analysis of all other moderators, those effect sizes would be combined using a weighted average procedure so that the study contributed only one effect size. This approach allowed us to retain as many data points from a study as possible, while minimizing any violations of independence assumptions.

Meta-analysis computation. For the calculation of the overall mean effect size, we chose to use the more commonly employed random-effects model (as recommended by Card, 2012; Cooper, 2009; Raudenbush, 2009). A random-effects model assumes that the effect sizes in the meta-analysis are randomly sampled from a population of effect sizes with a normal distribution and some average effect size. In this model, the variation seen in the sample of effect sizes used for the analysis comes both from sampling error 
and from systematic differences between studies (i.e., the model assumes that there is true variation in effect sizes). In contrast to random-effects models, fixed-effects models assume that there is only one true effect size and that variation in observed effect sizes is solely due to sampling error.

Random-effects models have a number of statistical and theoretical advantages over fixed-effect models. First, given the large variation in the specific manipulations and measures of construal level employed in our sample of studies (e.g., temporal distance manipulations that vary whether an event takes place next week vs. next month or today vs. thirty years from now), it seemed unrealistic to assume an identical true effect size across all studies. Second, the estimates of random-effects models are less affected by studies that are outliers either in terms of their effect sizes or in terms of the number of people in the study sample, due to a more even weighting of effect sizes across studies. Third, random-effects models generally have larger confidence intervals than fixedeffects models, also because of the way in which effect sizes are weighted in these models. Because of this, random-effects models provide a more conservative test of whether an average effect size is statistically significant or not, and therefore we can be more confident in our results. Finally, random-effects models allow for the results of a meta-analysis to be generalized beyond the particular studies used in the meta-analysis to the entire population of studies represented, due to the fact that random-effects models assume that the effect sizes used in a meta-analysis are a sample of the possible effect sizes sampled from a true population of effect sizes. A fixed-effect model, on the other hand, does not assume that the studies are sampled from a larger population of possible studies, and so the conclusions from a fixed-effect model cannot be generalized to studies 
not included in the meta-analysis. For all of the above reasons, we decided that a randomeffects model was the most appropriate choice for our analyses.

We implemented the random-effects model for the overall analyses, as well as the analyses for all categorical moderators, using the Comprehensive Meta-analysis 2 software. For all analyses of continuous moderators, we used the free meta-regression macro for SPSS developed by David Wilson, with a random-effects variance component based on the method of moment estimation (Lipsey \& Wilson, 2001). For moderator analyses, we used a mixed-effect model (i.e., treating the studies as a random sample to estimate the effect size in each group and then modeling the moderators as fixed effects; Card, 2012; Raudenbush, 2009). For categorical variables, we used a meta-analytic analogue of an ANOVA, which partitions the total variance into the portion explained by the potential moderator and the leftover variance that exists within levels of the moderator. The proportion of variance explained by the moderator is then tested against a chi-square distribution, the Q-test, for significance, with degrees of freedom equal to $\mathrm{j}-1$ where $\mathrm{j}$ reflects the number of levels of the categorical moderator. A significant Q-test indicates that there is significant moderation of the effect size (Card, 2012; Cooper, et al., 2009). For continuous moderators, we used a meta-regression, a form of a weighted regression (Card, 2012; Cooper et al., 2009).

\section{Direct Effect Results}

\section{Overall Analyses}

We identified 125 studies with a total of 310 effect sizes that met our inclusion criteria and measured a direct effect of psychological distance on construal level. The demographic information and observed effect sizes for each study can be found in the 
supplemental materials. The random-effects analysis yielded a mean effect size (Hedges' g) of $.475,95 \%$ CI $[.405, .545]$, which was significantly different from zero, $\mathrm{z}=13.31, p$ $<.001$, and which reflects a medium-sized effect according to the conventions set by Cohen (1988). As a comparison point, the average effect size found in social psychological research is $d=.430$ (Richard, Bond Jr., \& Stokes-Zoota, 2003). The effect sizes demonstrated a medium amount of heterogeneity, $\mathrm{I}^{2}=68.28 \%$, indicating that $68.28 \%$ of the total variation in effect sizes in our sample is due to true between-study variability, rather than sampling error (Higgins \& Thompson, 2002; Huedo-Medina, Sánchz-Meca, Martìn-Martìnez, \& Botella, 2006). This variability indicates the presence of moderating variables, which we return to explore shortly.

\section{Publication Bias}

We conducted a number of analyses to assess the accuracy of our effect size estimate. First, we calculated a fail-safe N, which is the number of studies with an average null effect size that would have to be added to the sample for the estimated average effect size to become non-significant (Rosenthal, 1979). This analysis yielded a fail-safe $\mathrm{N}$ of 15,202 studies, attesting to the robustness of the results.

In a meta-analysis, though, we care about not only the significance of an effect, but also its size. In order to assess whether our estimate of the mean effect size between psychological distance and construal level could be inflated due to publication bias, we constructed a funnel plot (see Figure 1), where the $\mathrm{x}$-axis represents the effect size and the y-axis represents the standard error of each study (Card, 2012). A funnel plot that is perfectly symmetric around the mean effect size would indicate that there is no evidence of publication bias. Unsurprisingly, given the prevalence of asymmetric funnel plots in 
psychology and the bias toward publishing significant results inherent in the current publication system (Bakker et a., 2012; Ferguson \& Brannick, 2012; Rosenthal, 1979), our funnel plot is not perfectly symmetric, indicating that our sample may be missing some studies with smaller effect sizes, leading to an overestimation of the true effect size.

To explore the extent to which publication bias may have inflated our effect size estimate, we performed a trim and fill analysis (Duval \& Tweedie, 2000). The trim and fill procedure imputes "missing" studies until symmetry is achieved, recalculating the overall effect size using the original sample and the imputed studies. When applied to our data, the procedure found 39 "missing" studies, and computed an overall effect size estimate of .299, 95\% CI [.226, .373]—still significant, but somewhat smaller than our unadjusted estimate.

The trim and fill procedure assumes that all funnel plot asymmetry is due to publication bias. However, in heterogeneous study samples, such as ours, funnel plot asymmetry can also reflect true differences in effect sizes between studies due to moderating variables (Sterne \& Egger, 2005; Terrin, Schmid, Lau, \& Olkin, 2003), as discussed below, in which case the effect size would not be overestimated. In the case of heterogenous samples, the trim and fill procedure can perform poorly (Terrin et al., 2003; Peters, Sutton, Jones, Abrams, \& Rushton, 2007), underestimating the true effect size. In other words, the funnel plot and trim and fill procedure here suggest a possible bias toward overestimation of the true effect size in our results, but could also reflect heterogeneity in effect sizes. Given that we cannot distinguish between them statistically, both possibilities bear consideration. 
Meanwhile, other biases can lead to underestimation of the true effect size (Card, 2012; Hunter \& Schmidt, 2004). In particular, unreliable measures lead to an attenuation in reported effect sizes, which means that a meta-analysis based on these attenuated results will underestimate the true size of the relation between the variables (Card, 2012; Hunter \& Schmidt, 2004; Schmidt, Le, \& Oh, 2009). Although techniques exist to attempt to correct for unreliability (e.g., Hunter \& Schmidt, 2004; Schmidt et al., 2009), we chose to allow the two potential opposing biases to help balance each other, with the aim of maximizing the accuracy of our estimates. Thus, in our analysis, the potential publication bias indicated by the funnel plot (which would lead to an overestimation of the effect size; Duval \& Tweedie, 2000) and the unreliability of measures we know to be present in the sample studies (which leads to an underestimation of the effect size; Bobko, Roth, \& Bobko, 2001, Card, 2012; Hunter \& Schmidt, 2004) are pushing the estimated average effect size in opposite directions. Though it is impossible to know which of these two biases is bigger, or whether they are of similar size to perfectly cancel each other out, they should counteract each other to some extent in terms of the mean effect size estimate. Taking these two counteracting forces and the large fail-safe $\mathrm{N}$ into account, we feel confident in concluding that there is a significant effect of psychological distance on construal level, and that our estimate of the effect size is as accurately centered as possible.

\section{Moderator Analyses}

As mentioned previously, we used a mixed-effects model to identify potential moderators of the effect of distance on construal level (see Card 2012). In a mixed-effects model, a random-effects model is used to estimate effect sizes and a fixed-effects model 
is used to test for moderation. For categorical moderators, we used an analogue of an ANOVA to test for moderation; for continuous moderators, we used an analogue of a weighted least squares regression analysis. Descriptive statistics for all moderators can be found in Table 3; confidence intervals and significance tests for each moderator are shown in Table 6.

Article characteristics. We tested whether three variables related to the characteristics of the articles moderated the size of the effect of distance on construal level, in order to explore the consistency of the effect across researchers, fields, and time. First, we investigated whether current or former members of the Trope and Liberman labs found the same effect sizes as researchers from independent labs. The analysis yielded a null effect, $Q(1)=.031, p=.869$, indicated that Trope and Liberman lab members did not find larger effects than other researchers. Indeed, the mean effect size was slightly higher for studies done by independent labs (.482 for independent labs versus .469 for researchers directly associated with the original authors of the theory).

Next, we tested whether studies published in journals outside of the fields of social psychology found different effect sizes from those published in social psychology journals. Given that construal level theory was originally developed within the field of social psychology but has since spread to a variety of other fields, we were interested to see how well the theory's predictions and methodologies have translated beyond its native area. This analysis also yielded a null effect, $Q(1)=.084, p=.772$, indicating that the effect size remains consistent across different fields.

Finally, we tested whether effect size was moderated by publication year. We did this in order to determine whether the effect size has declined over time, which can be a 
symptom of publication bias and which could indicate that the studies in the sample have overestimated the true mean effect size (Button et al., 2013; Schooler, 2011). Regressing effect size onto the year each study was published (or, in the case of unpublished studies, the year they were conducted) yielded no evidence that effect size changed across years, $Q(1)=.279, p=.598$. The stability of the effect size over time suggests that that sample of studies used in the present meta-analysis did not overestimate the true effect size of distance on construal level, nor did the initial studies conducted to test the theory. Across these three analyses, then, there is no evidence that the observed effect size depends on researcher, field, or time.

Sample characteristics. Next, we tested whether the effect of distance on construal level was moderated by three variables related to characteristics of the participant sample. First, we explored participant gender as a potential moderator, by testing whether the percentage of females in the study sample altered the size of the effect. A meta-regression analysis with the percentage of females in each study sample predicting effect size was not significant $Q(1)=1.52, p=.217 .^{4}$

Second, we examined whether the culture of the country in which the study was conducted altered the effect size of distance on construal level. A meta-regression with the effect size of each study regressed onto country-level individualism/collectivism scores was not significant, $Q(1)=.859, p=.354$, indicating the relation between

\footnotetext{
${ }^{4}$ For the sake of complete reporting, we note that testing the effect of gender composition using maximum likelihood rather than method of moment analysis yielded a marginal result, $p=.099$, due to the fact that the two analyses gave slightly different $\mathrm{T}^{2}$ estimates for this particular effect. However, given the lack of a clear theoretical explanation for such a pattern, the fact that it only emerged with one type of analysis and not the other, and the fact that no such pattern appeared in the indirect effects analysis reported below, this non-significant trend does not seem to warrant further discussion. (Note that in the rest of the analyses we report, method of moment and maximum likelihood always yielded similar results.)
} 
psychological distance and construal level did not differ depending on culture (at least as reflected in this country-level measure).

Finally, we examined whether studies performed in laboratory settings, in the field, or online yielded different effect sizes. Given that laboratory environments allow researchers greater control over the environment and can help minimize potential distractions, one might expect that effect sizes for laboratory studies would be generally higher than those for non-laboratory studies. Conversely, researchers often worry about the quality of online data, given that participants may be distracted by many other things in their environment (Sargis, Skitka, \& McKeever, 2013). Supporting the intuition that research setting can matter, this variable significantly moderated effect size, $Q(2)=$ 20.06, $p<.001$. Follow-up comparisons revealed that whereas studies conducted in laboratory or field settings did not produce different effect sizes, $Q(1)=.43, p=.512$, studies conducted online showed significantly smaller effect sizes than either laboratory $(Q(1)=18.57, p<.001)$ or field studies $(Q(1)=9.54, p=.002)$. The effect sizes for studies performed in person (either in the lab or in the field) were both significant, $p$ 's $<$ .05 , and those performed online were just shy of significant, $p=.057$, suggesting that psychological distance influences construal level in each setting. However, the smaller average effect size for online studies suggests that researchers may need to use larger samples when collecting data online.

Distance manipulation characteristics. We examined whether three variables related to the characteristics of the distance manipulation moderated the effect of psychological distance on construal level. We first examined whether portraying the distance manipulation to participants as real or imaginary moderated the effect of 
distance. Interestingly, distance manipulations yielded significantly larger effect sizes when the distance was portrayed as imaginary $(\mathrm{M}=.55, S E=.05)$ rather than real $(\mathrm{M}=$ $.35, S E=.03), Q(1)=10.74, p=.001$. Importantly, however, both real and imaginary distance manipulations yield medium-sized and highly significant effects, $p<.001$.

Second, we examined whether the four types of psychological distance (time, space, social distance, and hypotheticality) produce comparable effects on construal level. The analysis revealed no moderating effect of distance type, $Q(3)=3.79, p=.285$, indicating that the type of psychological distance did not significantly alter the size of a given manipulation's effect.

Finally, we examined whether future and past distance manipulations led to equivalent effects on construal level, for studies that manipulated temporal distance. There was no moderating effect of distance direction on effect size, $Q(1)=.94, p=.331$, indicating that future and past temporal distance manipulations had comparable effects on construal level.

Dependent variable characteristics. We examined whether the effect of distance on construal level differed depending on whether the dependent variable was perceptual or conceptual. There was no effect of measurement type, $Q(1)=.951, p=.330$, indicating that on conceptual and perceptual measures yielded comparable effect sizes.

Finally, in order to better specify the process by which distance influences construal level, we tested for differences in effect sizes between dependent variables that measured the processing of abstract information, concrete information, or the relative difference between the two. The analysis yielded a significant moderating effect, $Q(2)=$ $15.71, p<.001$, suggesting that effect sizes differed across these three categories of 
dependent variables. Follow-up comparisons revealed that whereas the effect of distance on dependent variables that assessed the processing of solely abstract versus solely concrete information did not differ from each other, $Q(1)=.170, p=.680$, effect sizes were significantly larger for dependent measures that assessed the relative difference in the processing of abstract versus concrete information rather than only one or the other, $Q(1)=12.83, p<.001$. The effect sizes for all three types of dependent variables were highly significant, $p$ 's $<.001$. Taken together, these results suggest that psychological distance changes the processing of both abstract and concrete information. Thus, measures that capture relative differences should tend to be more powerful than those that focus solely on either abstract or concrete information.

The shape of the relation between temporal distance and construal. One final moderator analysis of interest concerned the specifying the nature of the relation between temporal distance and construal level. As noted earlier, construal level theory posits that increasing distance will lead to relative changes in construal level-an event occurring in one month should be construed more abstractly than an event occurring in one week, and an event occurring in one year should be construed more abstractly than an event occurring in one month. Thus, in testing the theory, the vast majority of studies have only included two levels of distance (one that is relatively near and one that is relatively far). Because of this, research has yet to specify whether the relation between distance and construal level is linear (e.g., a unit increase in abstraction for each additional future day added), curvilinear (e.g., greater increases in abstraction as an event moves further away in time), or stepwise (e.g., a dichotomous distinction between concrete representations for events that feel "near" versus abstract representations for events that feel "far"). Indeed, 
whereas construal level theorists often assume a continuous relation between distance and construal level, other theories imply a more dichotomous perspective (e.g., Forster \& Dannenberg, 2010).

Because our meta-analysis aggregates across many different individual studies that have used various two-level manipulations of distance, it is ideally suited to help specify the shape of the relation between distance and construal level. We focus our analysis on temporal distance, both because manipulations of temporal distance can be compared using a common underlying unit (e.g., number of days), and because the large number of studies manipulating temporal distance provides us with an adequate sample for this type of analysis.

Assuming that there is a continuous relation between distance and construal level, the question of the specific shape of the relation is also an interesting one. Construal level theory itself does not specify what the shape of this relation should be, though some researchers have suggested that individuals should become less sensitive to changes in distance at more remote time points (e.g., the difference between tomorrow and the next day might be more powerful than the difference between 365 and 366 days; Maglio, Trope \& Liberman, 2013b; Zauberman, Kim, Malkoc, \& Betterman, 2009). On the other hand, one might also reason that as an object or event moves further into the future, an increasing number of intervening temporal landmarks could make it feel even further away (see e.g., Peetz \& Wilson, 2013). Such ideas suggest that distance could potentially have a curvilinear relation with construal level.

In a meta-analysis, although we cannot directly manipulate multiple levels of distance to test the shape of this relation, we can leverage the existing variability in the 
two-level manipulations used in past studies to indirectly estimate it. To do so, we regressed effect size on (1) the difference (in days) between the near and distant conditions of each study and (2) the distance of the near condition (the number of days from today for the time point specified in the near condition). Although our sample contained a handful of studies that specified a time difference between the near and far conditions of close to ten years (3650 days), the vast majority of the studies contained differences of one year or less. Thus, we restricted our analysis to only include temporal distance studies with a difference between levels of 365 days or less, to avoid extrapolating beyond our data.

A meta-regression equation with time difference (distant time point - near time point) and time1 (the near time point), both weighted mean centered, predicting effect size was significant, $\mathrm{Q}(2)=13.06, \mathrm{p}=.001$. There was a significant effect of time difference $(\mathrm{B}=.001, \mathrm{SE}=.001 \beta=.237, \mathrm{z}=2.19, p=.028)$, indicating that as the difference between near and far time points increased, studies produced larger effects. There was also a significant main effect of time1 $(\mathrm{B}=.021, \mathrm{SE}=.009, \beta=.271, \mathrm{z}=2.32$, $p=.021$ ), indicating that as the near time point moved further into the future, the study produced larger effects. ${ }^{5}$

\footnotetext{
${ }^{5}$ An outlier analysis using DFFIT and DFBETA statistics (Cohen, Cohen, West, \& Aiken, 2003) did not indicate any outliers that might be unduly influencing the regression results. Analyses using Studentized residuals did identify two potential outliers, but the results were not affected when these two points were excluded. A final analysis using the central leverage statistic identified three potential outliers. When these three studies (which had the three most distant-future time1 points) were removed, the time difference variable remained significant, $p=.012$, whereas the time 1 variable did not, $p=.228$, suggesting that more studies with distant-future time1 points would be useful for reliably estimating the time 1 effect. We also conducted two additional analyses to test the interaction between the near time point and the difference between time points and the significance of the square of the difference between near and far time points. A significant interaction would indicate that the effect of increasing the difference between the near and the far time point changed as that time difference moved further into the future. A significant quadratic term for time difference would indicate that a constant rate of increase in the time difference would not produce a corresponding constant increase in the effect size. Neither test reached significance, $(p=.268$ and $p=$
} 
These results give us information about the relation between starting time point, the size of the difference between near and far time points, and the effect size obtained by a given study: They suggest that studies will tend to find bigger effects of distance on construal level as the temporal distance manipulation moves further into the future (e.g., starting a week from now rather than a day from now) and as the distance between conditions increases (e.g., next week vs. next year as opposed to next week vs. next semester).

Translating these findings into their implications for the shape of the relation between distance and abstraction requires an additional inferential step — we need to move from thinking about the effect size (or the difference in abstraction between near and far conditions) to the level of abstraction one would expect to see at a single point in time (a day, a week, a month, etc.). In other words, the analysis described above gives us information about effect size (i.e., what is the predicted difference in construal level between a given near and distant time point?), whereas we want to understand the shape of the curve between temporal distance and construal level (as one increases, what happens to the other?).

We can take the inferential step required to translate from one question to the other by considering the following. Our results indicate that the effect of increasing the difference in distance between two levels of a temporal distance manipulation and the effect of moving the starting point (the near condition) further into the future are additive. In other words, each gives the resulting effect size an independent boost. When translated into their implications for our variables of interest, these two independent boosts for 
effect size imply that distance has a curvilinear relationship with abstraction (roughly akin to the shape sketched in Figure 2).

Consider a concrete example: Imagine a study that asks participants to think about an event occurring one week from today (near condition) versus two weeks from today (distant condition) — a difference of seven days. The difference in abstraction produced by these two time points, according to our regression equation, should be equal to a constant (the intercept) plus .091. Now imagine a study that used two weeks as its near time point and three weeks for its distant time point. Once again, the difference in time is seven days; however, because the near time point is now two weeks from today, the difference in abstraction between the two time points would be equal to the same constant plus .175. In other words, the same difference in future temporal distance (i.e., the same difference between two points along the x-axis in Figure 2) produces a larger difference in construal level (i.e., a larger difference between two points along the y-axis in Figure 2) as future temporal distance increases (i.e., as one moves from left to right along the $\mathrm{x}$ axis). Moreover, if a third study widened the gap between near and distant time points to two weeks (near condition) versus four weeks (distant condition), the difference in abstraction between the two time points would increase again. Thus, the results of our regression, when translated from effect size (difference scores) to absolute level of construal level (as depicted in Figure 2), imply that distance may have a continuous and curvilinear relation to construal level.

\section{Direct Effects Discussion}

Across 125 studies that included a total of 310 effects, we found a mean effect size of .475 for the effect of distance on level of construal, corresponding to a medium- 
sized effect according to the conventions set by Cohen (1988). There was significant variation in the effect sizes (approximately $68 \%$ of the variance was due to true betweenstudy variability, rather than sampling error), indicating the presence of moderating variables. We tested whether variables related to article, sample, distance manipulation, and dependent variable characteristics moderated this effect.

The results showed that the effect of distance on construal level was largely consistent across variations in when and where a given study was conducted. The effect size remained unchanged across year of publication, study authors, journal, gender composition of the sample, and culture. The one exception was an unsurprising result indicating that effect sizes were smaller in online studies - a pattern that could reflect the tendency for online studies to limit experimental control and increase distraction. Taken together, these results suggest that the effect of distance on construal level replicates across different times, places, and populations.

The effect of distance on abstraction was also similar across different types of psychological distance (temporal, spatial, social, and hypothetical). All four types of distance produced significant, medium-sized effects on construal level, supporting CLT's central prediction that variation along any dimension of psychological distance will influence level of abstraction.

One unexpected but potentially interesting findings emerged from our analyses. We found - perhaps contrary to researcher intuition - that manipulations of imagined distance produced larger effect sizes than manipulations of ostensibly real distance, though both types of manipulations produced highly significant effect sizes. We return to 
discuss possible explanations for this finding and to suggest some avenues for future empirical research later, in the General Discussion.

Our results also suggest that distance affects the processing of high- and low-level features to a similar extent. Construal level theory does not make specific predictions about how exactly distance changes construal; it simply proposes that increasing distance should lead people to construct relatively more abstract mental representations. The current findings therefore offer a novel and important insight into the specific nature of these representations: As distance increases, people construe objects less in terms of their low-level features and more in terms of their high-level features. Thus, whereas some researchers have posited that abstract features, because of their stability and germaneness across distance, should always be included in mental representations (Kim et al., 2009), the current findings suggest that in fact, representations can vary in their inclusion of both high-and low-level features. From a methodological perspective, these results also suggest that when possible, researchers should select dependent variables that capture the relative change in both high- and low-level features, since such measures showed greater effect sizes than those assessing changes in only high- or only low-level features.

Finally, our results suggest that the shape of the curve describing the relation between temporal distance and construal is curvilinear: Both the size of the difference between near and distant conditions and the distance of the near condition from today predict independent increases in effect sizes. This finding has important methodological implications, suggesting that researchers can increase the strength of their manipulations both by widening the gap in time between the near and distant future conditions in their study, as well as by moving that gap in time further into the future. Thus, a study 
measuring construal level should produce larger effect sizes if it asks participants to consider a policy that will be implemented next month versus next year than if it asks them to consider a policy that will be implemented tomorrow versus next month.

In addition to their methodological implications, these findings may also pave the way for future research examining the relations between objective distance, subjective distance, and construal. The curvilinear relation between (objective) temporal distance and construal level implied by our results could reflect something about the relation between objective and subjective distance (i.e., suggesting that felt temporal distance increases more rapidly than objective temporal distance), or something about the relation between subjective distance and construal level (i.e., suggesting that subjective distance influences abstraction in a nonlinear way), or both. Future empirical work could seek to distinguish these explanations by measuring subjective distance as well as construal level. We return to consider other future directions in the General Discussion.

\section{Downstream Consequence Results}

\section{Overall Analyses}

We identified 179 studies with a total of 426 effect sizes that met the inclusion criterion and measured the downstream consequences of construal level (i.e., predictions, evaluations, and behaviors). The supplemental materials include the demographic information and observed effect sizes for each study. The random-effects analysis yielded a mean effect size (Hedges' g) of .526, 95\% CI [.471, .582], which was significantly different from zero, $\mathrm{z}=18.69, \mathrm{p}<.001$, and reflects a medium-sized effect (Cohen, 1988). In addition, the effect size demonstrated a medium amount of heterogeneity, $\mathrm{I}^{2}=$ $73.17 \%$, indicating that $73.17 \%$ of the total variation in effect sizes in our sample is due 
to true between-study variability, rather than sampling error (Higgins \& Thompson, 2002; Huedo-Medina et al., 2006). The analyses for the downstream consequences are identical to those for the direct effects, except where noted.

\section{Publication Bias}

The fail-safe $\mathrm{N}$ was 47,106 , suggesting that the effect is quite robust: The effect would remain significant even if we had somehow missed more than 47,000 studies with an average null effect size. As with the direct effects, the current funnel plot (see Figure 3) was asymmetric, indicated a possible overestimation of the effect size. We again used the trim and fill procedure (Duval \& Tweedie, 2000) to investigate the possible extent of publication bias. The analysis found 59 "missing" studies, calculating an overall adjusted effect size of $.383,95 \%$ CI $[.33, .43]$.

As before, funnel plot asymmetry can also represent true variation in effect sizes (Sterne \& Egger, 2005; Terrin, Schmid, Lau, \& Olkin,2003), and the trim and fill procedure can perform poorly, underestimating the effect size in heterogeneous samples such as ours (Terrel et al., 2003; Peters et al., 2007). Additionally, as previously mentioned, any potential overestimation due to publication bias is offset to some extent by the underestimation due to the fact that we did not adjust our effect sizes for the unreliability of dependent measures. Thus, we suspect that our unadjusted estimate of the effect size is as accurately centered as possible, but these results should be interpreted as a range rather than one specific number - there is clearly a significant effect of distance on downstream consequences, and the unadjusted and adjusted confidence intervals provide us with a range of estimates about the size of that effect that capture the aggregated evidence currently provided by the literature. 


\section{Moderator Analyses}

As in the direct effect result section, we used a mixed-effects model to identify potential moderators of the effect of distance on downstream consequences of construal, using an ANOVA analogue for categorical moderators and a weighted least squares regression analogue for continuous moderators (Cooper et al., 2009). Descriptive statistics for all moderators can be found in Table 3; confidence intervals and significance tests for each moderator are shown in Table 7.

Article characteristics. An analysis comparing the effect sizes from studies done by current or former member of the Trope and Liberman labs to those performed by independent researchers yielded a null effect, $Q(1)=.007, p=.935$, indicating that, as with the direct effects, Trope and Liberman lab members did not find larger effects than other researchers.

An analysis comparing the effect sizes found in social psychology to those found in other fields in order to determine how well the theory's predictions and methodologies have translated beyond its field of origin showed a null effect $Q(1)=2.12, p=.145$, indicating that, as with direct effects, the effect size remained consistent across different fields.

A meta-regression analysis with the year of the study predicting downstream consequence effect sizes to test for a decline effect yielded a null result, $Q=.003, p=$ .958 , as in the direct effect analysis above. This lack of a decline effect suggests that the sample of studies in our meta-analysis did not overestimate the true effect size of distance on downstream consequences. The results of these three analyses are consistent with the 
idea that the observed effect size in the present meta-analysis is consistent across researchers, fields, and time.

Sample characteristics. Consistent with the direct effect results, a metaregression analysis with the percentage of females in each study sample predicting downstream consequence effect sizes yielded no effect of gender, $Q=.625, p=.429$, indicating that the effect of psychological distance on its downstream consequences did not differ depending on the proportion of females in the study sample.

A meta-regression with the downstream consequence effect size of each study regressed onto country-level individualism/collectivism scores yielded a null result, $Q=$ $.839, p=.360$, indicating that, as with the direct effects, the relation between psychological distance and its downstream consequences did not differ depending on the culture of the country in which the study took place.

Finally, mirroring the findings observed in the direct effect studies, there was a significant effect of study setting, $Q(2)=12.01, p=.002$. Follow-up analyses revealed that, as in the first meta-analysis, the effect sizes of studies performed in the field did not differ significantly from either those performed in the lab, $p=.773$. However, studies conducted online showed smaller effect sizes than both those conducted in the lab, $p<$ .001 , and those conducted in the field, $p=.035$. Importantly, all effect sizes were highly significant, $p$ 's $<.001$, indicating that psychological distance reliably influences downstream consequences of construal level in all three settings.

Distance manipulation characteristics. An analysis comparing real and imaginary distance manipulations revealed a marginally significant trend for distance manipulations to yield larger effect sizes when the distance was portrayed as imaginary 
$(\mathrm{M}=.56, S E=.03)$ rather than real $(\mathrm{M}=.47, S E=.04), Q(1)=3.07, p=.080$.

Interestingly, this pattern mirrors the significant finding seen in the direct effects metaanalysis, and we return to consider it in the General Discussion. Once again, both real and imaginary manipulations yield highly significant effect sizes, $p<.001$.

For the analysis of the type of distance as a potential moderator, hypothetical distance was not included, due to the fact that there were only four studies that met the inclusion criterion and manipulated hypothetical distance. Thus, there were not enough studies to accurately estimate and average effect size for hypothetical distance. The analysis comparing the effect sizes for manipulations of temporal, spatial, and social distance yielded a null result, $Q(2)=2.07, p=.355$, indicating that the type of psychological distance did not significantly alter the size of the effect on the downstream consequences of construal. This finding mirrors the results in the earlier meta-analysis of direct effects.

Dependent variable characteristics. We tested whether distance affected the three types of downstream consequences (evaluations, predictions, and behaviors) to the same extent. The analysis yielded a null effect, $Q(1)=1.21, p=.546$, indicating comparable effects of psychological distance on the three types of outcomes.

\section{Downstream Consequence Discussion}

Across 179 studies that included a total of 426 effect sizes, we found a mean effect size of .526 for the effect of distance on the downstream consequences of abstraction, which corresponds to a medium-sized effect. There was significant variation in the effect size (approximately $73 \%$ of the variance was due to true between-study variability, rather than sampling error), indicating the presence of moderating variables. 
We tested whether variables related to article, sample, distance manipulation, and dependent variable characteristics moderated this effect.

As in the analyses of the direct effect of distance on construal level described earlier, we found that the effect of distance on downstream consequences was largely consistent across variable related to article and sample characteristics. The effect size remained unchanged across year of publication, study authors, journal, gender composition, and the culture of the country in which the study was conducted. Additionally, we found that the effect size was consistent across the three types of psychological distance that could be included in this meta-analysis (temporal, spatial, and social) ${ }^{6}$ and the three types of downstream outcomes (evaluations, predictions and behaviors). Together, these findings indicate that the effect of distance on downstream consequences of abstraction replicates across different times, places, and populations, as well as across different distances and outcomes.

\section{General Discussion}

The present meta-analyses set out to determine the average effect size of psychological distance on abstraction and its downstream consequences, and to investigate potential moderators of these basic effects to shed new light on the phenomenon. Our analyses showed that changes in psychological distance had a significant and medium-sized effect on both abstraction and its downstream consequences. Both effects were highly stable across a variety of theoretically irrelevant variables, including author, publication area, year of publication, gender, and country

\footnotetext{
${ }^{6}$ Recall that because only four studies examined the effect of hypothetical distance on downstream consequences of abstraction, hypotheticality could not be included in this analysis.
} 
culture, indicating that the effects replicate across different times, samples, and researchers.

At the same time, there was a moderate amount of variability in the effect sizes overall, indicating the presence of moderating variables. Indeed, our analyses identified a number of specific moderators that have the potential to shed new light on how psychological distance and abstraction are related and point the way toward interesting areas for future research. For instance, in our meta-analysis of direct effect studies, we found that distance influences the processing of high and low level features to a similar extent. Because construal level theory focuses on the prediction that increasing distance should lead people to construct relatively more abstract representations, it has not yet specified the exact process by which these changes in abstraction come about. Our finding suggests that psychological distance changes construal by altering the processing of both high and low level features, providing an important initial step in understanding the process by which distance alters construal.

Our meta-analyses also identified an unexpected but interesting potential moderator: In both direct and downstream consequences studies, imagined (vs. ostensibly real) manipulations of distance showed larger effect sizes (this trend was significant in the direct effect studies and marginally significant in the downstream consequence studies). Because the same trend emerged across both analyses, we suspect that it reflects a real pattern rather than noise in the data, and therefore believe it deserves some discussion. In particular, one important difference between many of the real versus imaginary distance manipulations in our sample of studies is the extent to which they seem to thoroughly engage participants. For example, one typical imagined-distance 
manipulation asks participants to spend a few minutes thinking and writing about a day in their life at a near versus distant point in time (e.g., Wakslak, Nussbaum, Liberman, \& Trope, 2008). In contrast, many real-distance manipulations involve more subtly changing the date of an event-for instance, briefly noting that the stimuli in the dependent variable are pictures or current or former students at the university (Rim, Uleman, \& Trope, 2009). Imaginary (vs. ostensibly real) distance manipulations therefore seem likely to produce greater participant engagement with the experimental task.

The discussion above, although necessarily speculative, suggests the interesting possibility that the effects of psychological distance could be somewhat dependent on effort: The more fully participants engage with or inhabit the more remote (vs. near) point of distance, the larger the effect on construal may be. ${ }^{7}$ Though construal level theory itself does not speak to this possibility, there is at least one existing study suggesting that participants who engage in more thoughtful processing show larger effects of distance (Park, 2010). Thus, it may be that the more time or effort people spend in thinking about distant points, the more abstract their ensuing mental representations tend to be. After all, if the function of construal level is to enable mental travel across distance, then the more cognitively engaged a person is in attempting to traverse distance (e.g., planning for the future, communicating with a dissimilar other), the more they may tend to draw on and construct abstract representations. Such a hypothesis might prove to be a fruitful direction for future research. Additionally, the notion that effortful processing enhances the effect of distance on construal level complements previous

\footnotetext{
${ }^{7}$ Note that we are not claiming that the effect requires mental elaboration-only that elaboration may enhance the effect.
} 
research distinguishing abstraction from heuristic processing (see e.g., Fujita, Eyal, Chaiken, Liberman, \& Trope, 2008; Ledgerwood, Trope, \& Chaiken, 2010).

Finally, our analyses suggest that temporal distance and abstraction have a curvilinear relationship. This finding has important methodological implications for researchers seeking to maximize the strength of their manipulations, and lays a foundation for future empirical research to examine the relation between objective and subjective distance and the relation between subjective distance and construal level.

Such research could help to resolve the intriguing discrepancy between the shape of the curve implied by our results on the one hand, and the opposite curve suggested by studies on preference reversal and time discounting, which show decreased sensitivity to changes in distance as distance increases (e.g., Maglio, Trope, \& Liberman, 2013a; Zauberman et. al, 2009). The latter body of work suggests that people see a larger difference between today and tomorrow, for instance, than they do between 364 and 365 days in the future. Importantly, such studies tend to involve presenting participants with both time points (e.g., asking participants to choose between a certain amount of money today or more money tomorrow). In contrast, the vast majority of the studies used in our analysis involve between-subjects designs that present participants with only a single time point (e.g., a policy that will be implemented next year). When participants compare two time points in a within-subjects design, they consider not only how far both time points are from the present, but also the proportional difference between the two time points (i.e., the relative difference in time between the two points). For example, receiving two dollars two days from now, rather than one dollar tomorrow, requires waiting twice as long. However, receiving two dollars in 366 days, rather than one dollar 
365 days, only requires waiting 1.003 times as long. Thus, the relative difference between the two times points is much larger in the near than in the distant future, and as such may have a larger effect on behavior. In a between-subjects design, this relative difference information is not available to participants, since they only see a single time point. Instead, they simply consider the absolute distance of the near and far time points from the present. Thus, it may be that within-subjects designs show how perceptions of relative differences in time affect behavior, whereas between-subjects designs show how changes in absolute distance affect behavior. If future empirical work supports this difference, it would imply that researchers employing within-subjects designs may wish to keep their two time points closer to the present, whereas researchers using a between-subjects design may wish to move their two time points further into the future in order to maximize the strength of the manipulation.

It is also important to note that our analysis was restricted in range (since nearly all past studies have used manipulations within the range of 0 to 365 days) and that it was necessarily correlational. We therefore cannot rule out potential confounds that may have varied along with the temporal distance manipulations selected by researchers. Thus, though these findings provide an intriguing, initial perspective on the potential relation between distance and construal, further experimental work that includes multiple time conditions is clearly warranted.

One other limitation of this meta-analysis is that we were not able to simultaneously include all potential moderators in one meta-regression, due to the fact that the structure of our data necessitated a shifting unit of analysis approach. Thus, one might wonder whether our results would differ if we tested our moderators together, 
rather than one at a time. There is in fact a newer method for dealing with dependent effect sizes called the correlated effects method (Hedges, Tipton, \& Johnson, 2010; see Footnote 2), which enabled us to run a meta-regression that included most (though not all) of our moderators simultaneously. Importantly, the results of this meta-regression were extremely similar to the results of our individual ANOVAs - that is, each of the effects reported above also emerged using this alternative analysis. In other words, there was no evidence from this meta-regression that any of the effects we found were due to our moderators being confounded with each other. We therefore feel confident in concluding that the results reported here are not an artifact of the particular analytic strategy we employed.

\section{Future Directions}

Taken together, then, our findings help to shed light on a number of interesting questions while also highlighting some areas of research on distance and abstraction that clearly merit further attention. First, although a large number of experiments have tested the effects of psychological distance on abstraction and its downstream consequences, the vast majority of studies manipulate temporal distance, and future temporal distance in particular. Researchers could do more to explore other types of distance that have been less well studied thus far - most notably, past temporal distance and hypotheticality. Likewise, past research has focused far more on conceptual than perceptual measures of construal level, and more on evaluative downstream consequences than behavioral ones. Additional research involving the less well studied types of distance and outcome variables would enable more precise estimates of the effect sizes for these study parameters. 
Second, to date, very little work has examined boundary conditions of the effects of psychological distance on abstraction. Nearly all existing empirical work has been concerned with showing how distance affects abstraction across a variety of domains, but very little research has investigated when or for whom this relation may not hold, or may be smaller or larger in magnitude (for a recent and important exception, see Fujita, Darwent, Cheavens, and Lazarus, in preparation). Our meta-analyses suggest some variables that may moderate the size of this relationship, but clearly, more empirical work is needed.

Relatedly, little empirical work has been conducted to help specify the nature of the process by which psychological distance lead to changes in the abstractness of mental representation. To date, most CLT research has focused on abstraction in mental representation as the process by which distance can affect evaluation, prediction, and behavior. A critical next step is to zero in on the process underlying the link between distance and mental representation itself. A handful of existing studies have begun to shed some light on this question, suggesting that distance can affect early visual processing of more abstract or concrete stimuli, activity in sensory-motor regions of the brain, and explicit weights placed on feasibility versus desirability concerns (e.g., Amit, Mehoudar, Trope, \& Yovel, 2012; Gilean, Liberman, \& Maril, 2013; Liviatan, Trope, \& Liberman, 2008). Other plausible processes include attention, accessibility, and memory for high- and low-level features. For instance, if a person is forming a representation of a new clock radio by reading a description that contains both high- and low-level information, one possibility is that distance affects the mental representation she constructs of the radio by altering the type of information that she attends to while 
reading the description, thus changing the content that is included in the representation itself (e.g., if low-level information is not attended to and encoded, it may not be stored as part of the mental representation of the toaster). Another possibility is that she initially attends to both types of information, but distance leads her to assign greater importance to one type of information over the other, thereby producing a more structured mental representation. A third possibility is that distance could affect what type of information about the clock radio is more accessible in memory. In the latter two examples, the information about the radio contained in the mental representation is the same, but distance alters how that content is further processed and used to make decisions about the toaster. Although our findings suggest that distance produces changes in both high- and low-level features, they do not and cannot specify the process by which this occurs. We believe that a high priority for future research will be to further clarify the processes underlying the effect of psychological distance on mental representation.

Finally, several interesting questions remain that fell outside the scope of the present meta-analyses. First, the question of how multiple manipulations of distance and/or construal might combine when encountered together is intriguing, given that in real life, objects often vary along several distance dimensions at once (e.g., an event that will take place in a nearby location at a distant future point in time). Although a few studies have combined manipulations of two types of distance or distance and a procedural prime of construal level (e.g., Fujita, Eyal, Chaiken, Trope, \& Liberman, 2008, Study 3; Maglio, Trope, \& Liberman, 2013), the effects of such combinations on abstraction have not been systematically studied to determine the exact nature of their interaction and what factors, if any, will alter the way in which they interact. 
Second, construal level theory hypothesizes that the link between distance and construal is bidirectional: Changes in psychological distance should affect construal level and changes in construal level should affect perceptions of psychological distance. The present meta-analyses focused on the first direction in this link as an important — and sizeable - first step in elucidating the relation between distance and construal level. As the number of studies testing the reverse direction grows, a meta-analysis synthesizing those studies will become an important next step.

\section{Conclusion}

Taken together, the findings of our meta-analyses indicate that psychological distance has a medium-sized effect on both construal level and the downstream consequences of abstraction, including a wide range of consequential outcomes such as political preferences, negotiation, and consumer behavior. The effects of psychological distance are consistent across a diverse set of researchers and research conditions, suggesting that they have broad generalizability. The results also suggest several moderators that have important theoretical and methodological implications. Future research should further explore the boundary conditions of the effect of psychological distance on abstraction as well as the specific cognitive mechanisms that underlie this effect. Such work could build on the meta-analytic results presented here to continue expanding and refining our understanding of how humans are able both to immerse themselves within immediate experience as well as to transcend it. 


\section{References}

References marked with an astericks indicate studies included in the meta-analyses.

* Agerström, J., \& Bjorklund, F. (2009a). Moral concerns are greater for temporally distant events and are moderated by value strength. Social Cognition, 27, 261-282. doi:10.1521/soco.2009.27.2.261.

* Agerström, J., \& Bjorklund (2009b). Temporal distance and moral concerns: Future morally questionable behavior is perceived as more wrong and evokes stronger prosocial intentions. Basic and Applied Social Psychology, 31, 49-59. doi:10.1080/0197353080265988.

* Agerström, J., Bjorklund, F., \& Allwood, M. (2010). The influence of temporal distance on justiceand care motives. Scandinavian Journal of Psychology, 51, 46-55. doi:10.1111/j.1467-9450.2009.00724.x.

* Agerström, J., Bjorklund, F., \& Carlsson, R. (2012). Emotions in time: Moral emotions appear more intense with temporal distance. Social Cognition, 30, 181-198. doi:10.1521/soco.2012.30.2.181.

* Amit, E., Algom, D., \& Trope, Y. (2009). Distance-dependent processing of pictures and words. Journal of Experimental Psychology: General, 138, 400-415. doi:10.1037/a0015835.

Amit, E., Mehoudar, E., Trope, Y., \& Yovel, G. (2012). Do object-category selective regions in the ventral visual stream represent perceived distance information? Brain and Cognition, 80, 201-213. doi:10.1016/j.bandc.2012.06.006. 
* Amit, E., Wakslak, C. J., \& Trope, Y. (2013). The use of visual and verbal means of communication across psychological distance. Personality and Social Psychology Bulletin, 39, 43-56. doi:10.1177/0146167212460282.

Badre, D., \& D’Esposito, M. (2007). Functional magnetic resonance imaging evidence for a hierarchical organization of the prefrontal cortex. Journal of Cognitive Neuroscience, 19, 2082-2099. doi:10.1162/jocn.2007.19.12.2082.

* Bar-Anan (2009). The effect of temporal distance on the behavior identification form: a replication. Unpublished data.

Bakker, M., van Dijk, A., \& Wicherts, J. M. (2012). The rules of the game called psychological science. Perspectives on Psychological Science, 7, 543-554. doi:10.3758/s13428-011-0089-5

Borenstein, M. (2009). Effect Sizes For Continuous Data. In H. Cooper, L. V. Hedges, \& J. C. Valentine (Eds.), The handbook of research synthesis and meta-analysis (2nd ed., pp. 317-333). New York: Russel Sage Foundation.

Borenstein, M., Hedges, L., Higgins, J., \& Rothstein, H. (2005). Comprehensive MetaAnalysis (Version 2) [Computer Software]. Englewood, NJ: Biostat.

Bobko, P., Roth, P., \& Bobko, C. (2001). Correcting the effect size of d for range and unreliability. Organizational Research Methods, 4, 46-61.

* Borovoi, L., Liberman, N., \& Trope, Y. (2010). The effects of attractive but unattainable alternatives on the attractiveness of near and distant future menus. Judgment and Decision Making, 5, 102-109. 
Burgoon, E. M., Henderson, M. D., \& Markman, A. B. (2013). There are many ways to see the forest for the trees: A tour guide for abstraction. Perspectives on Psychological Science, 8, 501-520.

* Burgoon, E. M., Henderson, M.D., \& Wakslak, C. J. (2013). How do we want others to decide?: Geographical distance influences evaluations of decision makers. Personality and Social Psychology Bulletin, 39, 826-838. doi:10.1177/0146167213481247.

* Burrus, J., \& Roese, N. J. (2006). Long ago it was meant to be: The interplay between time, construal, and fate beliefs. Personality and Social Psychology Bulletin, 32, 1050-1058. doi:10.1177/0146167206288282.

Button, K. S., Ioannidis, J. P. A., Mokrysz, C., Nosek, B. S., Flint, J., Robinson, E. S. J., \& Munafò, M. R. (2013). Power failure: why small sample size undermines the reliability of neuroscience. Nature Reviews Neuroscience, 14, 1-12.

Card, N. A. (2012). Applied meta-analysis for social science research. New York: Guilford.

* Carter, S. E., \& Sanna, L. J. (2008). It's not just what you say but when you say it: Selfpresentation and temporal construal. Journal of Experimental Social Psychology, 44, 1339-1345. doi:10.1016/j.jesp.2008.03.017.

Chan, M. E., \& Arvey, R. D. (2012). Meta-analysis and the development of knowledge. Perspectives on Psychological Science, 7, 79-92. doi:10.1177/1745691611429355.

* Chiu, F. (2012). Fit between future thinking and future orientation on creative imagination. Thinking Skills and Creativity, 7, 234-244. doi:10.1016/j.tsc.2012.05.002. 
* Choi, S. Y., Park, H. S., \& Oh, J. Y. (2011). Temporal distance and blood donation intentions. Journal of Health Psychology, 17, 590-599. doi: $10.1177 / 1359105311421048$.

* Chou, H., \& Lien, N. (2012). The effect of incentive types and appeal regulatory framing in travel advertising. The Service Industries Journal, 32, 883-897. doi: $10.1080 / 02642069.2010 .545878$.

* Christian, B. M., Miles, L. K., Fung, F.H. K., Best, S., \& Macrae, C. N. (2013). The shape of things to come: Exploring goal-directed prospection. Consciousness and Cognition, 22, 471-478. doi: 10.1016/j.concog.2013.02.002.

Clark, H. H., \& Brennan, S. E. (1991). Grounding in communication. Perspectives on Socially Shared Cognition, 13, 127-149.

Cohen, J. (1988). Statistical power analysis for the behavioral sciences (2nd ed.). New York, NY: Psychology Press.

Cohen, J., Cohen, P., West, S. G., \& Aiken, L. S. (2003). Applied multiple regression/correlation analysis for behavioral sciences, $3^{\text {rd }}$ Edition. Routledge.

* Conway, P., \& Peetz, J. (2012). When does feeling moral actually make you a better person? Conceptual abstraction moderates whether past moral deeds motivate consistency or compensatory behavior. Personality and Social Psychology Bulletin, 38, 907-919. doi:10.1177/0146167212442394.

* Cojuharenco, I., Patient, D., \& Bashur, M.R. (2011). Seeing the "forest" or the "trees" of organizational justice: Effects of temporal perspective on employee concerns about unfair treatment at work. Organizational Behavior and Human Decision Processes, 116, 17-31. doi:10.1016/j.obhdp.2011.05.008. 
Cooper, H. (2009). Research synthesis and meta-analysis: A step-by-step approach. (4th ed). Thousand Oaks, CA: Sage.

Cooper, H., Hedges, L. V., \& Valentine, J. C. (Eds). (2009). The handbook of research synthesis and meta-analysis. 2nd Edition New York: Russell Sage Foundation, 2009.

* Danziger, S., Monit, R., \& Barkan, R. (2012). Idealistic advice vs. pragmatic choice: A psychological distance account. Journal of Personality and Social Psychology, 102, 1105-1117. doi:10.1037/a0027013.

* Darwent, K. M. (2012). Individual differences in travel across psychological distances (Unpublished doctoral dissertation). The Ohio State University.

* Day, S. B., \& Bartels, D. M. (2008). Representations over time: The effect of temporal distance on similarity. Cognition, 106, 1504-1513. doi:10.1016/j.cognition.2007.05.013.

* De Dreu, C. K.W., Giacomantonio, M., Shalvi, S., \& Sligte, D. (2009). Getting stuck or stepping back: Effect of obstacles and construal level in the negotiation of creative solutions. Journal of Experimental Social Psychology, 45, 542-548. doi:10.1016/j.jesp.2009.01.001.

Ekstrom, R. B., French, J. W., Harman, H. H., \& Dermen, D. (1976). Kit offactorreferenced cognitive tests. Princeton, NJ: Educational Testing Services.

* Eyal, T., Hoover, G.M., Fujita, K., \& Nussbaum, S. (2011). The effect of distancedependent construals on schema-driven impression formation. Journal of Experimental Social Psychology, 47, 278-281. doi:10.1016/j.jesp.2010.10.007. 
* Eyal, T., Liberman, N., \& Trope, Y. (2008). Judging near and distance virtue and vice. Journal of Experimental Social Psychology, 44, 1204-1209. doi:10.1016/j.jesp.2008.03.012.

* Eyal, T., Liberman, N.,Trope, Y., \& Walther, E. (2004). The pros and cons of temporally near and distant action. Journal of Personality and Social Psychology, 86, 781-795. doi:10.1037/0022-3514.86.6.781.

* Eyal, T., Sagristano, M. D., Trope, Y., Liberman, N., \& Chaiken, S. (2009). When values matter: Expressing values in behavioral intentions for the near vs. distant future. Journal of Experimental Social Psychology, 45, 35-43. doi:10.1016/j.jesp.2008.07.023

Ferguson, C. J., \& Brannick, M. T. (2012). Publication bias in psychological science: Prevalence, methods for identifying and controlling, and implications for the use of meta-analyses. Psychological Methods, 17, 120-128. doi:10.1037/a0024445.

* Förster, J. (2009). Relations between perceptual and conceptual score: How global versus local processing fits a focus on similarity versus dissimilarity. Journal of Experimental Psychology: General, 138, 88-111. doi:10.1037/a0014484.

* Förster, J., \& Becker, D. (2012). When curiosity kills no cat—but mediates the relation between distant future thoughts and global processing across sensory modalities. European Journal of Social Psychology, 42, 334-341. doi:10.1002/ejsp.1856

Förster, J., \& Dannenberg, L. (2010). GLOMO sys: A systems account of global versus local processing. Psychological Inquiry, 21, 175-197. doi:10.1080/1047840X.2010.487. 
* Förster, J., Friedman, R. S., \& Liberman, N. (2004). Temporal construal effects on abstract and concrete thinking: Consequences for insight and creative cognition. Journal of Personality and Social Psychology, 87, 177-189. doi:10.1037/00223514.87.2.177

* Freitas, A. L., Salovey, P., \& Liberman, N. (2001). Abstract and concrete selfevaluative goals. Journal of Personality and Social Psychology, 80, 410-424. doi:10.1037//0022-3514.80.3.410.

* Freitas, A. L., Langsam, K. L., Clark, S., \& Moeller, S. J. (2008). Seeing oneself in one's choices: Construal level and self-pertinence of electoral and consumer decisions. Journal of Experimental Social Psychology, 44, 1174-1179. doi:10.1016/j.jesp.2008.02.011.

Fujita, K., Darwent, K. M., Cheavens, J. S., \& Lazarus, S. (in prep). Depressive symptomology and the association between abstraction and temporal distance: A preliminary test of the functional benefits of abstraction in mental time travel.

* Fujita, K., Eyal, T., Chaiken, S., Trope, Y., \& Liberman, N. (2008). Influencing attitudes toward near and distant objects. Journal of Experimental Social Psychology, 44, 562-572. doi:10.1016/j.jesp.2007.10.005.

* Fujita, K., Henderson, M., Eng, J., Trope, Y., \& Liberman, N. (2004).Unpublished data.

* Fujita, K., Henderson, M., Eng, J., Trope, Y., \& Liberman, N. (2006). Spatial distance and mental construal of social events. Psychological Science, 17, 278-282.

doi:10.1111/j.1467-9280.2006.0. 
* Fukukura, J., Ferguson, M., \& Fujita, K. (2013). Psychological distance can improve decision making under information overload via gist memory. Journal of Experimental Psychology: General, 142, 658-665. doi:10.1037/a0030730.

* Garcia, D. (2011). Happy today, happy tomorrow: The (non-) effect of temporal distance on judgments of life satisfaction. Personality and Individual Differences, 51, 1048-1051. doi:10.1016/j.paid.2011.07.031.

* Garcia, D., Rosenberg, P., \& Siddiqui, A. (2011). Tomorrow I could be in trouble...but the sun will come out next year: The effect of temporal distance on adolescent's judgments of life satisfaction. Journal of Adolescence, 34, 751-757. doi:10.1016/j.adolescence.2010.08.006.

* Giacomantonio, M., De Dreu, C. K. W., \& Mannetti, L. (2010). Now you see it, now you don't: Interests, issues, and psychological distance in integrative negotiation. Journal of Personality and Social Psychology, 98, 761-774. doi:10.1037/a0017879.

* Giacomantonio, M., De Dreu, C. K. W., Shalvi, S., Sligte, D., \& Leder, S. (2010). Psychological distance boosts value-behavior correspondence in ultimatum bargaining and integrative negotiation. Journal of Experimental and Social Psychology, 46, 824-829. doi:10.1016/j.jesp.2010.05.001.

* Gilead, M., Liberman, N., \& Maril, A. (2012). Construing counterfactual worlds: The role of abstraction. European Journal of Social Psychology, 42, 391-397. doi:10.1002/ejsp.1862

* Gong, H., \& Medin, D. L. (2012). Construal level and moral judgment: Some complications. Judgment and Decision Making, 7, 628-638. 
* Goodman, J. K., \& Malkoc, S. A. (2012). Choosing here and now versus there and later: The moderating role of psychological distance on assortment size preference. Journal of Consumer Research, 39, 751-768. doi:10.1086/665047.

* Halamish, V., Nussinson, R., \& Ben-Ari, L. (2013). In a year, memory will benefit from learning, tomorrow it won't: Distance and construal level effects on the basis of meta-memory judgments. Journal of Experimental Psychology: Learning, Memory, and Cognition, 39, 1621-1627. doi:10.1037/a0032381.

Hedges, L. V., Tipton, E., \& Johnson, M. C. (2010). Robust variance estimation in metaregression with dependent effect size estimates. Research Synthesis Methods, 1, 3965. doi:10.1002/jrsm.5.

* Heller, D., Stephan, E., Kifer, Y., \& Sedikides, C. (2011). What will I be? The role of temporal perspective in predictions of affect, traits, and self-narratives. Journal of Experimental Social Psychology, 47, 610-615. doi:10.1016/j.jesp.2011.01.010.

* Henderson, M. (2009). Psychological distance and group judgments: The effect of psychological distance on believes about common goals. Personality and Social Psychological Bulletin, 35, 1330-1341. doi:10.1177/0146167209340905.

* Henderson, M. (2011). Mere physical distance and integrative agreement: When more space improves negotiation outcomes. Journal of Experimental Social Psychology, 47, 7-15. doi:10.1016/j.jesp.2010.07.011.

* Henderson, M., Fujita, K., Trope, Y., \& Liberman, N. (2006). Transcending the "here": The effect of spatial distance on social judgment. Journal of Personality and Social Psychology, 91, 845-856. doi:10.1037/0022-3514.91.5.845. 
* Henderson, M., Trope, Y., \& Carnevale, P. J. (2006). Negotiation from a near and distance time perspective. Journal of Personality and Social Psychology, 91, 712729. doi:10.1037/0022-3514.91.4.712.

* Henderson, M. D., \& Wakslak, C. J. (2010). Psycholgical distance and priming: When do semantic primes impact social evaluations? Personality and Social Psychology Bulletin, 36, 975-985. doi:10.1177.0146167210367490.

* Herzog, S. M., Hansen, J., \& Wankem M. (2007). Temporal distance and ease of retrieval. Journal of Experimental Social Psychology, 43, 483-488. doi:10.1016/j.jesp.2006.05.008.

Higgins, J. P. T., \& Thompson, S. G. (2002). Quantifying heterogeneity in a metaanalysis. Statistics in Medicine, 21, 1539-1558.

Hofstede, G., Hofstede, G. J., \& Minkov, M. (2010). Cultures and organizations: Software of the mind (3rd ed.). McGraw-Hill: USA.

Huedo-Medina, T. B., Sánchz-Meca, J., Martìn-Martìnez, F., \& Botella, J. (2006). Assessing heterogeneity in meta-analysis: Q statistic or $\mathrm{I}^{2}$ index? Psychological Methods, 11, 193-206. doi:1037/1082-989X.11.2.193.

Hunter, J. E., \& Schmidt, F. L. (2004). Methods of meta-analysis: Correcting error and bias in research findings (2nd ed.). Thousand Oaks, CA: Sage.

Ioannidis, J. P. A. (2005). Why most published research findings are false. PLos Med, 2. * Janakiraman, N., \& Ordonez, L. (2012). The effect of effort and deadlines on consumer product returns. Journal of Consumer Psychology, 22, 260-271. doi:10.1016/j.jcps.2011.05.002. 
* Jia, L., Hirt, E. R., \& Karpen, S. C. (2009). Lessons from a faraway land: The effect of spatial distance on creative cognition. Journal of Experimental Social Psychology, 45, 1127-1131. doi:10.1016/j.jesp.2009.05.015.

* Jia, L., \& Smith, E. R. (2013). Distance makes the metaphor grow stronger: A psychological distance model of metaphor use. Journal of Experimental Social Psychology, 49, 492 - 497. doi:10.1016/j.jesp.2013.01.009.

* Jiga-Boy, G. M., Clark, A. E., \& Semin, G. R. (2013). Situating construal level: The function of abstractness and concreteness in social contexts. Social Cognition, 31, 201-221. doi:10.1521/soco.2013.31.2.201.

* Jin, L., \& He, Y. (2013). Designing service guarantees with construal fit: Effects of temporal distance on consumer responses to service guarantees. Journal of Service Research, 16, 202-215. doi:10.1177/1094670512468330.

* Kane, J., van Boven, L., \& McGraw, P. (2012). Prototypical prospective: Future events are more prototypically represented and simulated than past events. European Journal of Social Psychology, 42, 354-362. doi:10.1002/ejsp.1866.

* Kim, Y., Park, J, \& Wyer Jr., R. S. (2009). Effects of temporal distance on memory and consumer judgments. Journal of Consumer Research, 36, 634-645. doi:10.1086/599765.

* Kivetz, Y., \& Tyler, T. R. (2007). Tomorrow I'll be me: The effect of time perspective on the activation of idealistic versus pragmatic selves. Organizational Behavior and Human Decision Processes, 102, 193-211. doi:10.1016/j.obhdp.2006.07.002. 
Kühnen, U., \& Oyserman, D. (2002). Thinking about the self influences thinking in general: Cognitive consequences of salient self-concept. Journal of Experimental Social Psychology, 38, 492-499. doi:10.1016/S0022-1031(02)000.

* Leach, F. R., \& Plaks, J. E. (2009). Regret for errors of commission and omission in the distant term versus near term: The role of level of abstraction. Personality and Social Psychology Bulletin, 35, 221-229. doi: 10.1177/0146167208327001.

* Ledgerwood, A., \& Callahan, S. P. (2010). Distance modulates susceptibility to local versus global social influences. Unpublished Data. University of California, Davis.

* Ledgerwood, A, Trope, Y., \& Chaiken, S. (2010). Flexibility now, consistency later: Psychological distance and construal shape evaluative responding. Journal of Personality and Social Psychology, 99, 32-51. doi:10.1037/a0019843.

* Ledgerwood, A., \& Wakslak, C. J. (2007). Differential information use for near and distant decisions: Additional Experiment. Unpublished Data. New York University.

* Ledgerwood, A., Wakslak, C. J., \& Wang, M. A. (2010). Differential information use for near and distant decisions. Journal of Experimental Social Psychology, 46, 638642. doi:10.1016/j.jesp.2010.03.001.

Lehrer, J. (2010). The truth wears off. The New Yorker. p. 52.

Leslie, A. M. (1987). Pretense and representation: The origins of "theory of mind." Psychological Review, 94, 412.

* Liberman, N., \& Forster, J. (2009). The effect of psychological distance on perceptual level of construal. Cognitive Science, 33, 1330-1341. doi:10.1111/j.15516709.2009.01061.x. 
* Liberman, N., Sagristano, M., \& Trope, Y. (2002). The effect of temporal distance on level of mental construal. Journal of Experimental Social Psychology, 38, 523-534. doi:10.1016/S0022-1031(02)00535-8.

* Liberman, N., \& Trope, Y. (1998). The role of feasibility and desirability considerations in near and distant future decisions: A test of temporal construal theory. Journal of Personality and Social Psychology, 75, 5-18. doi:10.1037/00223514.75.1.5.

Liberman, N., \& Trope, Y. (2008). The psychology of transcending the here and now. Science, 332, 1201-1205. doi:10.1126/science.1161958.

Lipsey, M. W., \& Wilson, D. B. (2001). Practical meta-analysis. Thousand Oaks, CA: Sage.

* Liviatan, I., Trope, Y., \& Liberman, N. (2008). The effect of similarity on mental construal. Journal of Experimental Social Psychology, 44, 1256-1269. doi:10.1016/j.jesp.2008.04.007.

* Liviatan, I., Trope, Y., \& Liberman, N. A construal level analysis of the similarityattraction relation. Unpublished Data, New York University.

* Lu, J., Xie, X., \& Xu, J. (2012). Desirability and Feasibility: Self-other decisionmaking differences. Personality and Social Psychology Bulletin, 39, 144-155. doi:10.1177/0146167212470146

* Lutchyn, Y., \& Yzer, M. (2011). Construal level theory and theory of planned behavior: Time frame effects on salient belief generation. Journal of Health Communication, 16, 595-606. doi:10.1080/10810730.2011.551991. 
* Maglio, S. J., \& Trope, Y. (2011). Scale and construal: How larger measurement units shrink length estimates and expand mental horizons. Psychonomic Bulletin Review, 18, 103-109. doi:10.3758/s13423-010-0025-1.

Maglio, S. J., Trope, Y., \& Liberman, N. (2013a). Distance from a distance: Psychological distance reduces sensitivity to any further psychological distance. Journal of Experimental Psychology: General, 142, 644-657. doi:10.1037/a0030258.

Maglio, S. J., Trope, Y., \& Liberman, N. (2013b). The common currency of psychological distance. Current Directions in Psychological Science, 22, 278-282. doi:10.1177/0963721413480172.

* Malkoc, S. A., Zauberman, G., \& Ulu, C. (2005). Consuming now or later? The interactive effect of timing and attribute alignability. Psychological Science, 16, 411417. doi:10.111/j.0956-7976.2005.01549.x.

* McCathy, R. J., \& Skowronski, J. J. (2011). You're getting warmer: Level of construal affects the impact of central traits on impression formation. Journal of Experimental Social Psychology, 47, 1304-1307. doi:10.1016/j.jesp.2011.05.017.

* McGraw, A. P., Warren, C., Lawrence, W. E., \& Leonard, B. (2012). Too close for comfort, or too far to care? Finding humor in distant tragedies and close mishaps. Psychological Science, 23, 1215-1223. doi:10.1177/0956797612443831.

Medin, D. L. (1989). Concepts and conceptual structure. American Psychologist, 44, 1469-1481. doi:10.1037/0003-066X.44.12.1469.

Menegatti, M., \& Rubini, M. (2013). Convincing similar and dissimilar others: The power of language abstraction in political communication. Personality and Social Psychology Bulletin, 39, 596-607. doi:10.1177/0146167213479404. 
* Milkman, K. L., Modupe, A., \& Chugh, D. (2012). Temporal distance and discrimination: An audit study in academia. Psychological Science, 23, 710-717. doi:10.1177/0956797611434539.

Michel, W., Shoda, Y., \& Rodriguez, M. I. (1989). Delay of gratification in children. Science, 244, 933-938.

Nisbett, R. E., Peng, K., Choi, I., \& Norenzayan, A. (2001). Culture and systems of thought: Holistic versus analytic cognition. Psychological Review, 108, 291-300. doi:10.1037/0033-295X.108.2.291.

* Nussbaum, S., Liberman, N., \& Trope, Y. (2006). Predicting the near and distant future. Journal of Experimental Psychology: General, 135, 152-161. doi:10.1037/00963445.135.2.152.

* Nussbaum, S., Trope, Y., \& Liberman, N. (2003). Creeping disposition: The temporal dynamic of behavior prediction. Journal of Personality and Social Psychology, 84, 485-497. doi:10.1037//0022-3514.84.3.485.

* Park, K. (2010). The role of elaboration moderating the effects of temporal construal on evaluation. Seoul Journal of Business, 16, 65-92.

* Park, L. E., Young, A. F., \& Eastwick, P. W. (under review). Psychological distance makes the heart grow fonder: Effects of psychological distance and relative intelligence on men's attraction to women.

* Peetz, J., \& Buehler, R. (2012). When distance pays off: The role of construal level in spending predictions. Journal of Experimental Social Psychology, 48, 395-398. doi:10.1016/j.jesp.2011.07.016. 
* Peetz, J., Buehler, R., \& Wilson, A. (2010). Planning for the near and distance future: How does temporal distance affect task completion predictions? Journal of Experimental Social Psychology, 46, 709-720. doi:10.1016/j.jesp.2010.03.008.

Peetz, J., \& Wilson, A. E. (2013). Post-birthday world: Consequences of temporal landmarks for temporal self-appraisal and motivation. Journal of Personality and Social Psychology, 104, 249-266. doi:10.1037/a0030477.

Perugini, M., Gallucci, M., \& Constantini, G. (2014). Safeguard power as a protection against imprecise power estimates. Perspectives on Psychological Science, 9, 319 332. doi:10.1177/1745691614528519.

Peters, J. L., Sutton, A. J., Jones, D. R., Abrams, K. R., \& Rushton, L. (2008). Contour enhanced funnel plots help distinguish publication bias from other causes of asymmetry. Journal of Clinical Epidemiology, 61, 991-996.

doi:10.1016/j.jclinepi.2007.11.010.

* Plaks, J. E., McNichols, N. K., \& Fortune, J. L. (2009). Thoughts versus deeds: Distal and proximal intent in lay judgments of moral responsibility. Personality and Social Psychology Bulletin, 35, 1687-1701. doi:10.1177/0146167209345529.

* Polman, E., \& Emich, K. J. (2011). Decisions for others are more creative than decisions for the self. Personality and Social Psychology Bulletin, 37, 492-501. doi:10.1177/0146167211398362.

Raudenbush, S. W. (2009). Analyzing Effect Sizes: Random-Effects Models. In H. Cooper, L. V. Hedges, \& J. C. Valentine (Eds.), The handbook of research synthesis and meta-analysis (2nd ed., pp. 317-333). New York: Russel Sage Foundation. 
Richard, F. D., Bond Jr., C. F., Stokes-Zoota, J. J. (2003). One hundred years of social psychology quantitatively described. Review of General Psychology, 7, 331-363. doi:10.1037/1089-2680.7.4.331.

* Rim, S., Hansen, J., \& Trope, Y. (2013). What happens why? Psychological distance and focusing on causes versus consequences of events. Journal of Personality and Social Psychology, 104, 457-472. doi:10.1037/a0031024.

* Rim, S., Uleman, J. S., \& Trope, Y. (2009). Spontaneous trait inference and construal level theory: Psychological distance increases nonconscious trait thinking. Journal of experimental social psychology, 45, 1088-1097. doi:10.1016/j.jesp.2009.06.015.

Rosch, E., Mervis, C. B., Gray, W. D., Johnson, D. M., \& Boyes-Braem, P. (1976). Basic objects in natural categories. Cognitive Psychology, 8, 382-439. doi:10.1016/00100285(76)90013-X.

* Roehm, M. L., \& Roehm Jr., H. A. (2011). The influence of redemption time frame on responses to incentives. Journal of Academic Marketing Science, 39, 363-375. doi:10.1007/s11747-010-0201-X.

* Rogers, Y., \& Bazerman, M. H. (2008). Future lock-in: Future implementation increases selection of 'should' choices. Organizational Behavior and Human Decision Processing, 106, 1-20. doi:10.1016/j.obhdp.2007.08.001.

Rosenthal, R. (1979). The "file drawer problem" and tolerance for null results. Psychological Bulletin, 86, 638-641. doi:10.1037/0033-2909.86.3.638.

* Sanchez, A. M., \& Ledgerwood, A. (2012a). Unpublished data.

* Sanchez, A. M., \& Ledgerwood, A. (2012b). Unpublished data. 
* Sanchez, A. M., \& Rutchick, A. M. (2013). Distance, prevention, and resilience in decisions about national security risks. Poster presented at the $14^{\text {th }}$ annual Society for Personality and Social Psychology in New Orleans, LA.

* Schimmel, K., \& Forster, J. (2008). How temporal distance changes novices' attitudes towards unconventional art. Psychology of Aesthetics, Creativity, and the Arts, 2, 5360. doi:10.1037/1931-3896.2.1.53.

Schmidt, F. L., Le, H., \& Oh, I. S. (2009). Correcting for the distorting effects of study artifacts in meta-analysis. In H. Cooper, L. V. Hedges, \& J. C. Valentine (Eds.), The handbook of research synthesis and meta-analysis (2nd ed., pp. 317-333). New York: Russel Sage Foundation.

Schooler, J. W., (2011). Unpublished results hide the decline effect. Nature, 470, 437.

Semin, G. R., \& Fielder, K. (1988). The cognitive functions of linguistic categories in describing persons: Social cognition and language. Journal of Personality and Social Psychology, 131, 364-376.

Shacter, D. L. (2012). Adaptive constructive processes and the future of memory. American Psychologist, 67, 603-613.

* Steinhart, Y., Carmon, Z., \& Trope, Y. (2013). Distant warnings of adverse side-effects can backfire. Psychological Science, 24, 1842-1847.

* Steinhart, Y., Mazursky, D., \& Kamins, S. (2013). The "temporal-processing-fit effect": The interplay between regulatory state, temporal distance, and construal level. Social Cognition, 31, 315-335. doi:10.1521/soco.2013.31.3.315. 
* Stephan, E., Liberman, N., \& Trope, Y. (2010). Politeness and psychological distance: A construal level perspective. Journal of Personality and Social Psychology, 98, 268280. doi:10.1037/a0016960.

Sterne, J. A. C., \& Egger, M. (2005). Regression methods to detect publication and other bias in meta-analysis. In H. R. Rothstein, A. J. Simon \& M. Borenstein (Eds.), Publication bias in meta-analysis. Prevention, assessment and adjustments (pp. 99110). Hoboken, NJ: Wiley.

Suddenorf, T., \& Corballis, M. C. (2007). The evolution of foresight: What is mental time travel and is it unique to humans? Behavioral and Brain Science, 30, 299-313.

Tam, L., Glassman, M., \& Vanderwauver, M. (2010). The psychology of password management: A tradeoff between security and convenience. Behaviour and Information Technology, 29, 233-244. doi:10.1080/01449290903121386.

Tanner-Smith, E., \& Tipton, E. (in press). Robust variance estimation with dependent effect sizes: Practical considerations and a software tutorial in Stata and SPSS. Research Synthesis Methods.

Terrin, N., Schmid, C. H., Lau, J., \& Olkin, I. (2003). Adjusting for publication bias in the presence of heterogeneity. Statistics in Medicine, 22, 2113-2126. doi:10.1002/sim.1461.

* Thomas, M., Chandran, S., \& Trope, Y. (2006). The effects of information type and temporal distance on purchase intentions. Unpublished Data.

Tipton, E. (in press). Small sample adjustments for robust variance estimation with metaregression. Psychological Methods. 
* Todorov, A., Goren, A., \& Trope, Y. (2007). Probability as a psychological distance: Construal and preferences. Journal of Experimental Social Psychology, 43, 473-482. doi:10.1016/j.jesp.2006.04.002.

* Trope, Y., \& Liberman, N. (2000). Temporal construal and time-dependent changes in preference. Journal of Personality and Social Psychology, 79, 876-889. doi:10.1037/0022-3514.79.6.876.

Trope, Y., \& Liberman, N. (2003). Temporal Construal. Psychological Review, 110, 403421. doi:10.1037/0033-295X.110.3.403.

Trope, Y., \& Liberman, N. (2010). Construal-level theory of psychological distance. Psychological Review, 117, 440-463. doi:10.1037/a0018963.

Vallacher, R. R., \& Wegner, D. M. (1987). What do people think they're doing? Action identification and human behavior. Psychological Review, 94, 3-15. doi:10.107/0033295X.94.1.3.

Vallacher, R. R., \& Wegner, D. M. (1989). Levels of personal agency: Individual variation in action identification. Journal of Personality and Social Psychology, 57, 660-671. doi:10.1037/002-3514.57.4.660.

* Volk, S., \& Korner, A. Temporal distance increases utilitarian decisions in the crying baby dilemma. Unpublished Manuscript.

* von Wagner, C., Semmler, C., Power, E., \& Good, A. (2010). What matters when deciding whether to participate in colorectal cancer screening? The moderating role of time perspective. Journal of Applied Biobehavioral Research, 15, 20-30. doi:10.1111/j.1751-9861.2010.00050.x. 
* Wakslak, C. J., Nussbaum, S., Liberman, N., \& Trope, Y. (2008). Representations of the self in the near and distant future. Journal of Personality and Social Psychology, 95, 757-773. doi:10.1037/a0012939.

* Wakslak, C. J., Trope, Y., Liberman, N., \& Alony, R. (2006). Seeing the forest when entry is unlikely: Probability and the mental representation of events. Journal of Experimental Psychology: General, 135, 641-653. doi:10.1037/0096-3445.135.4.641.

Walton, G. M., Cohen, G. L., Cwir, D., \& Spencer, S. J. (2012). Mere belonging: The power of social connections. Journal of Personality and Social Psychology, 102, 513532. doi:10.1037/a0025731.

* Warner, R. H., Vandeursen, M. J., \& Pope, A. R. D. (2012). Temporal distance as a determinant of just world strategy. European Journal of Social Psychology, 42, 276284. doi:10.1002/ejsp.1855.

*Wyer, N. A. (2014). Unpublished raw data.

* Wyer, N. A., Perfect, T. J., \& Pahl, S. (2010). Temporal distance and person memory: Thinking about the future changes memory for the past. Personality and Social Psychology Bulletin, 36, 805-816. doi:10.1177/0146167210370965.

* Yan, D., \& Sengupta, J. (2011). Effects of construal level on the price-quality relationship. Journal of Consumer Research, 38, 376-389. doi:10.1086/659755.

* Yan, D., \& Sengupta, J. (2013). The influence of base rate and case information on health-risk perceptions: A unified model of self-positivity and self-negativity. Journal of Consumer Research, 39, 931-946. doi:10.1086/666596.

Yong, E. (2012). Replication studies: Bad copy. Nature, 485, 298-300. 
Zauberman, G., Kim, B. K., Malkoc, S. A., \& Bettman, J. R. (2009). Discounting time and time discounting: Subjective time perception and intertemporal preference. Journal of Marketing Research, 46, 543-556. doi:10.1509/jmkr.46.4.543. 
CONSTRUAL LEVEL META-ANALYSIS

Table 1

Examples of more abstract (higher-level) versus concrete (lower-level) characteristics

\begin{tabular}{|l|l|}
\hline Higher-level & Lower-level \\
\hline Desirability concerns & Feasibility concerns \\
Broad categories & Exemplars or narrow categories \\
Gestalts & Details \\
Words & Pictures \\
Primary features & Secondary features \\
Broad traits & Specific behaviors \\
Dispositional information & Situational information \\
Aggregate information & Individualized information \\
Overarching goals, values, or ideologies & Situatio n-specific demands \\
\hline
\end{tabular}


Table 2

\section{Example psychological distance manipulations}

\begin{tabular}{|c|c|}
\hline Distance Type & Manipulation \\
\hline Temporal & $\begin{array}{l}\text { Participants imagine their life tomorrow vs. a year from now } \\
\text { and write about it for five minutes } \\
\text { - Participants imagine making a choice tomorrow vs. a month } \\
\text { from now } \\
\text { - Participants make predictions about events that will occur in } \\
\text { the near or distant future }\end{array}$ \\
\hline Spatial & $\begin{array}{l}\text { Participants imagine they are going on a trip to a nearby or } \\
\text { distant location } \\
\text { - Participants are told that the study materials were created at a } \\
\text { nearby or distant location } \\
\text { - Participants believe they are talking to or making judgments } \\
\text { about others who are in a nearby or distant location }\end{array}$ \\
\hline Social & $\begin{array}{l}\text { Participants are asked to make a choice for themselves or for } \\
\text { another person } \\
\text { - Participants make judgments about a similar (same birthday) } \\
\text { or dissimilar (different birthday) individual } \\
\text { - Participants made judgments about ingroup or outgroup } \\
\text { members }\end{array}$ \\
\hline Hypothetical & $\begin{array}{l}\text { - Participants believe there is a high or low likelihood that they } \\
\text { will complete a task later in the study } \\
\text { - Participants make judgments about an event that has a high } \\
\text { or low probability of occurring }\end{array}$ \\
\hline
\end{tabular}


Table 3

List of moderator variables for direct effect and downstream consequence analyses with their operationalizations and descriptive statistics

\begin{tabular}{|c|c|c|c|c|}
\hline \multirow[b]{2}{*}{ Moderator } & \multirow[b]{2}{*}{ Value } & \multirow[b]{2}{*}{ Coding Description and Criteria } & \multicolumn{2}{|c|}{ Descriptive Statistics } \\
\hline & & & Direct Effects & Downstream Consequences \\
\hline \multicolumn{5}{|c|}{ Article Characteristics } \\
\hline Original Lab & $\mathrm{N}=\mathrm{No}$ & Categorical variable representing whether any author of & $\mathrm{k}=125$ & $\mathrm{k}=179$ \\
\hline & $\mathrm{Y}=\mathrm{Yes}$ & the paper is/was a member of the original theorists' labs. & $\begin{array}{l}\text { No } k=62 \\
\text { Yes k }=63\end{array}$ & $\begin{array}{l}\text { No } k=93 \\
\text { Yes } k=86\end{array}$ \\
\hline Psychology & $\mathrm{N}=\mathrm{No}$ & Categorical variable representing whether the article was & $\mathrm{k}=115$ & $\mathrm{k}=169$ \\
\hline Journal & $\mathrm{Y}=\mathrm{Yes}$ & $\begin{array}{l}\text { published in a journal within the field of social/cognitive } \\
\text { psychology or not. }\end{array}$ & $\begin{array}{l}\text { No } k=10 \\
\text { Yes } k=105\end{array}$ & $\begin{array}{l}\text { No } k=40 \\
\text { Yes } k=129\end{array}$ \\
\hline Year & Continuous & $\begin{array}{l}\text { Continuous variable representing the year the study was } \\
\text { published. For unpublished studies, it represents the year } \\
\text { the research was conducted. }\end{array}$ & $\begin{array}{l}\mathrm{k}=120 \\
\mathrm{M}=2008.92 \\
\mathrm{SD}=3.26 \\
\text { Range }=1998-2013\end{array}$ & $\begin{array}{l}\mathrm{k}=175 \\
\mathrm{M}=2009.35 \\
\mathrm{SD}=3.32 \\
\text { Range }=1998-2013\end{array}$ \\
\hline \multicolumn{5}{|c|}{ Sample Characteristics } \\
\hline $\begin{array}{l}\text { Percentage } \\
\text { Female }\end{array}$ & Continuous & $\begin{array}{l}\text { Continuous variable representing the proportion of } \\
\text { females in the study sample. }\end{array}$ & $\begin{array}{l}\mathrm{k}=98 \\
\mathrm{M}=64.16, \mathrm{SD}=13.93 \\
\text { Range }=18-100\end{array}$ & $\begin{array}{l}\mathrm{k}=136 \\
\mathrm{M}=62.69, \mathrm{SD}=14.83 \\
\text { Range }=0-100\end{array}$ \\
\hline Culture & Continuous & $\begin{array}{l}\text { Continuous variable representing the } \\
\text { individualism/collectivism of the country in which the } \\
\text { study was performed. }\end{array}$ & $\begin{array}{l}\mathrm{k}=118 \\
\mathrm{M}=75.68 \\
\mathrm{SD}=20.06 \\
\text { Range }=17-91\end{array}$ & $\begin{array}{l}\mathrm{k}=166 \\
\mathrm{M}=71.34 \\
\mathrm{SD}=24.29 \\
\text { Range }=17-91\end{array}$ \\
\hline Setting & $\begin{array}{l}0=\text { Lab } \\
1=\text { Field } \\
2=\text { Online }\end{array}$ & $\begin{array}{l}\text { Categorical variable representing whether the study was } \\
\text { performed in a laboratory setting or a non-laboratory } \\
\text { setting. }\end{array}$ & $\begin{array}{l}\mathrm{k}=125 \\
\text { Lab } \mathrm{k}=106 \\
\text { Field } \mathrm{k}=6 \\
\text { Online } \mathrm{k}=13\end{array}$ & $\begin{array}{l}\mathrm{k}=182 \\
\text { Lab } \mathrm{k}=127 \\
\text { Field } \mathrm{k}=27 \\
\text { Online } \mathrm{k}=25\end{array}$ \\
\hline \multicolumn{5}{|c|}{ Manipulation Characteristics } \\
\hline $\begin{array}{l}\text { Real or } \\
\text { Imagined }\end{array}$ & $\begin{array}{l}\mathrm{R}=\text { Real } \\
\mathrm{I}=\text { Imagined }\end{array}$ & $\begin{array}{l}\text { Categorical variable representing whether the } \\
\text { manipulation of distance in the study was perceived by } \\
\text { the participants to be real or imaginary. }\end{array}$ & $\begin{array}{l}\mathrm{k}=125 \\
\text { Real } \mathrm{k}=39 \\
\text { Imagined } \mathrm{k}=86\end{array}$ & $\begin{array}{l}\mathrm{k}=181 \\
\text { Imagined } \mathrm{k}=110 \\
\text { Real } \mathrm{k}=71\end{array}$ \\
\hline
\end{tabular}




\begin{tabular}{|c|c|c|c|c|}
\hline Distance type & $\begin{array}{l}0=\text { Temporal } \\
1=\text { Spatial } \\
2=\text { Social } \\
3=\text { Hypothetical }\end{array}$ & $\begin{array}{l}\text { Categorical variable representing the type of } \\
\text { psychological distance that was manipulated in the } \\
\text { study. }\end{array}$ & $\begin{array}{l}\mathrm{k}=121 \\
\text { Temporal } \mathrm{k}=84 \\
\text { Spatial } \mathrm{k}=12 \\
\text { Social } \mathrm{k}=17 \\
\text { Hypothetical } \mathrm{k}=8\end{array}$ & $\begin{array}{l}\mathrm{k}=179 \\
\text { Temporal } \mathrm{k}=132 \\
\text { Spatial } \mathrm{k}=20 \\
\text { Social } \mathrm{k}=23 \\
\text { Hypothetical } \mathrm{k}=4\end{array}$ \\
\hline Past or Future & $\begin{array}{l}P=\text { Past } \\
F=\text { Future }\end{array}$ & $\begin{array}{l}\text { Categorical variable representing whether the temporal } \\
\text { distance manipulation was in the past or the future. }\end{array}$ & $\begin{array}{l}\mathrm{k}=87 \\
\text { Future } \mathrm{k}=78 \\
\text { Past } \mathrm{k}=9\end{array}$ & N/A \\
\hline Near Time & Continuous & $\begin{array}{l}\text { Continuous variable representing the number of days } \\
\text { from the present the near condition was. For studies that } \\
\text { gave a duration (e.g., "during this week"), we used the } \\
\text { midpoint of the duration (i.e., } 3.5 \text { days). }\end{array}$ & $\begin{array}{l}\mathrm{k}=53 \\
\mathrm{M}=4.52, \mathrm{SD}=7.43 \\
\text { Range }=0-31\end{array}$ & N/A \\
\hline Far Time & Continuous & $\begin{array}{l}\text { Continuous variable representing the number of days } \\
\text { from the present the far condition was. For studies that } \\
\text { gave a duration (e.g., during this week), we used the } \\
\text { midpoint of the duration (i.e., } 3.5 \text { days). }\end{array}$ & $\begin{array}{l}\mathrm{k}=53 \\
\mathrm{M}=276.51, \mathrm{SD}=129.17 \\
\text { Range }=7-365\end{array}$ & N/A \\
\hline $\begin{array}{l}\text { Time } \\
\text { difference }\end{array}$ & Continuous & $\begin{array}{l}\text { Continuous variable representing the difference in days } \\
\text { between the near and far conditions (only computed for } \\
\text { studies in which the far condition did not exceed } 365 \\
\text { days). }\end{array}$ & $\begin{array}{l}\mathrm{k}=53 \\
\mathrm{M}=271.99, \mathrm{SD}=127.58 \\
\text { Range }=7-364\end{array}$ & N/A \\
\hline $\begin{array}{l}\text { Dependent Variab } \\
\text { Perceptual or } \\
\text { Conceptual }\end{array}$ & $\begin{array}{l}\text { Characteristics } \\
\mathrm{P}=\text { Perceptual } \\
\mathrm{C}=\text { Conceptual }\end{array}$ & $\begin{array}{l}\text { Categorical variable representing whether the dependent } \\
\text { variable was perceptual or conceptual. }\end{array}$ & $\begin{array}{l}\mathrm{k}=130 \\
\text { Perceptual }=20 \\
\text { Conceptual }=110\end{array}$ & N/A \\
\hline $\begin{array}{l}\text { Focus of } \\
\text { Measure }\end{array}$ & $\begin{array}{l}\text { AH }=\text { High } \\
\text { Level } \\
\mathrm{AL}=\text { Low Level } \\
\mathrm{R}=\text { Relative }\end{array}$ & $\begin{array}{l}\text { Categorical variable representing whether the dependent } \\
\text { variable assessed only changes in high-level } \\
\text { characteristics, only low-level characteristics, or the } \\
\text { relative change in both. }\end{array}$ & $\begin{array}{l}\mathrm{k}=169 \\
\text { Relative }=95 \\
\text { High }=33 \\
\text { Low }=41\end{array}$ & N/A \\
\hline $\begin{array}{l}\text { Type of } \\
\text { Outcome }\end{array}$ & $\begin{array}{l}1=\text { Evaluation } \\
2=\text { Prediction } \\
3=\text { Behavior }\end{array}$ & $\begin{array}{l}\text { Categorical variable representing whether the dependent } \\
\text { variable measured an evaluation, prediction, or behavior. }\end{array}$ & NA & $\begin{array}{l}\mathrm{k}=177 \\
\text { Evaluation } \mathrm{k}=111 \\
\text { Prediction } \mathrm{k}=30 \\
\text { Behavior } \mathrm{k}=36\end{array}$ \\
\hline
\end{tabular}


CONSTRUAL LEVEL META-ANALYSIS

Table 4

Examples of dependent variables that measure high-level, low-level, or relative change in mental representations

\begin{tabular}{|l|l|}
\hline $\begin{array}{l}\text { Low-level } \\
\text { indicators }\end{array}$ & $\begin{array}{l}\text { 1. Stated interest in receiving secondary information } \\
\text { 2. The number of cons generated during a writing task } \\
\text { 3. The number of links to feasibility information selected for viewing }\end{array}$ \\
\hline $\begin{array}{l}\text { High-level } \\
\text { indicators }\end{array}$ & $\begin{array}{l}\text { 1. Stated interest in receiving primary information } \\
\text { 2. The number of pros generated during a writing task } \\
\text { 3. The number of links to desirability information selected for viewing }\end{array}$ \\
\hline $\begin{array}{l}\text { Relative } \\
\text { indicators }\end{array}$ & $\begin{array}{l}\text { 1. Relative interest in receiving primary versus secondary information } \\
\text { 2. The proportion of pros to cons generated during a writing task } \\
\text { 3. Relative preference for describing actions in terms of concrete } \\
\text { means versus abstract ends (the Behavioral Identification Form) }\end{array}$ \\
\hline
\end{tabular}


CONSTRUAL LEVEL META-ANALYSIS

Table 5

Example evaluation, prediction, and behavior dependent variables

\begin{tabular}{|c|l|}
\hline Outcome Type & \multicolumn{1}{c|}{ Example Dependent Variable } \\
\hline \multirow{3}{*}{ Evaluation } & $\bullet$ Liking for a product \\
\cline { 2 - 2 } & $\bullet$ Satisfaction with a negotiation offer \\
\cline { 2 - 2 } & $\bullet$ Interest in an activity \\
\hline \multirow{3}{*}{ Prediction } & $\bullet$ Anticipated guilt \\
\cline { 2 - 2 } & $\bullet$ Risk assessment \\
\cline { 2 - 2 } & $\bullet$ Predicted task completion time \\
\hline \multirow{3}{*}{ Behavior } & $\bullet$ Voting intention \\
\cline { 2 - 2 } & $\bullet$ Amount of time volunteered \\
\cline { 2 - 2 } & $\bullet$ Number of raffle tickets purchased \\
\hline
\end{tabular}


CONSTRUAL LEVEL META-ANALYSIS

Table 6

Moderator analyses for direct effect analyses.

\begin{tabular}{|c|c|c|c|c|c|c|}
\hline Moderator & $\mathrm{k}$ & ES & $\mathrm{SE}$ & $95 \% \mathrm{CI}$ & $\begin{array}{l}\text { Test statistic: } \\
\mathrm{Q}(\mathrm{df}) \text { or } z\end{array}$ & $\begin{array}{c}p \\
\text { value }\end{array}$ \\
\hline \multicolumn{7}{|l|}{ Article } \\
\hline \multicolumn{7}{|l|}{ Characteristics } \\
\hline Original Lab & & & & & $.031(1)$ & .860 \\
\hline No & 62 & .482 & .054 & {$[.38, .59]$} & & \\
\hline Yes & 63 & .469 & .048 & {$[.38, .56]$} & & \\
\hline Psychology Journal & & & & & $.084(1)$ & .772 \\
\hline No & 10 & .555 & .168 & {$[.23, .88]$} & & \\
\hline Yes & 105 & .505 & .034 & {$[.44, .57]$} & & \\
\hline Year & 120 & $\mathrm{~B}=-.006$ & .011 & {$[-.03, .02]$} & $.279(1)$ & .597 \\
\hline \multicolumn{7}{|l|}{ Sample } \\
\hline \multicolumn{7}{|l|}{ Characteristics } \\
\hline Percentage Female & 98 & $\mathrm{~B}=.004$ & .003 & {$[-.002, .01]$} & $1.52(1)$ & .217 \\
\hline Culture & 118 & $\mathrm{~B}=.002$ & .002 & {$[-.002, .01]$} & $.859(1)$ & .354 \\
\hline Setting & & & & & $12.29(2)$ & .001 \\
\hline $\mathrm{Lab}$ & 106 & .511 & .038 & {$[.44, .56]$} & & \\
\hline Field & 6 & .597 & .125 & {$[.35, .84]$} & & \\
\hline Online & 13 & .145 & .076 & {$[.00, .29]$} & & \\
\hline \multicolumn{7}{|l|}{ Manipulation } \\
\hline \multicolumn{7}{|l|}{ Characteristics } \\
\hline Real or Imagined & & & & & $10.74(1)$ & .001 \\
\hline Imagined & 86 & .551 & .052 & {$[.45, .65]$} & & \\
\hline Real & 39 & .349 & .034 & {$[.28, .42]$} & & \\
\hline Distance type & & & & & $3.79(3)$ & .285 \\
\hline Temporal & 84 & .493 & .046 & {$[.40, .58]$} & & \\
\hline Spatial & 12 & .315 & .083 & {$[.15, .48]$} & & \\
\hline Social & 17 & .467 & .070 & {$[.33, .61]$} & & \\
\hline Hypotheticality & 8 & .500 & .095 & {$[.31, .68]$} & & \\
\hline Past or Future & & & & & $.944(1)$ & .331 \\
\hline Past & 9 & .407 & .101 & {$[.21, .60]$} & & \\
\hline Future & 78 & .516 & .050 & {$[.42, .61]$} & & \\
\hline Near Time & 53 & $\mathrm{~B}=.021$ & .009 & {$[.003, .038]$} & $\mathrm{z}=2.32$ & .021 \\
\hline
\end{tabular}


Time Difference

$53 \mathrm{~B}=.001 \quad .001 \quad[.000, .002] \quad \mathrm{z}=2.19 \quad .028$

Dependent

Variable

Characteristics

Perceptual or

Conceptual

Perceptual

Conceptual

$20 \quad .404 \quad .085 \quad[.24, .57]$

$\begin{array}{llll}110 & .495 \quad .039 \quad[.42, .57]\end{array}$

Focus of Measure

High Level

$\begin{array}{llll}33 & .349 & .054 & {[.24, .46]} \\ 41 & .321 & .043 & {[.24, .40]} \\ 95 & .558 & .047 & {[.47, .65]}\end{array}$

$15.71(2) \quad<.001$

Low Level

$.951(1)$

.330

Relative

Note. Means and standard deviations reported for continuous variables are unweighted

for ease of interpretation, but were weighted in all analyses. 
Table 7

Moderator analyses for downstream consequence analyses. We suggest using $C t r l+F$ and keyword to find specific effects of interest.

\begin{tabular}{|c|c|c|c|c|c|c|}
\hline Moderator & $\mathrm{k}$ & ES & SE & $95 \% \mathrm{CI}$ & $\begin{array}{c}\text { Test } \\
\text { statistic: } \\
\mathrm{Q}(\mathrm{df}) \text { or } z\end{array}$ & $\begin{array}{c}p \\
\text { value }\end{array}$ \\
\hline \multicolumn{7}{|l|}{$\begin{array}{l}\text { Article } \\
\text { Characteristics }\end{array}$} \\
\hline Original Lab & & & & & $.007(1)$ & .935 \\
\hline No & 93 & .522 & .037 & {$[.45, .59]$} & & \\
\hline Yes & 86 & .527 & .041 & {$[.45, .61]$} & & \\
\hline Psychology Journal & & & & & $2.12(1)$ & .145 \\
\hline No & 40 & .605 & .056 & {$[.50, .71]$} & & \\
\hline Yes & 129 & .512 & .032 & {$[.45, .57]$} & & \\
\hline Year & 175 & $\mathrm{~B}=.004$ & .008 & {$[-.02, .02]$} & $.003(1)$ & .958 \\
\hline \multicolumn{7}{|l|}{ Sample } \\
\hline Characteristics & & & & & & \\
\hline Percentage Female & 136 & $\mathrm{~B}=.001$ & .002 & {$[-.002, .005]$} & $.626(1)$ & .429 \\
\hline Culture & 166 & $\mathrm{~B}=-.001$ & .001 & {$[-.003, .001]$} & $.839(1)$ & .360 \\
\hline Setting & & & & & $12.01(2)$ & $<.001$ \\
\hline $\mathrm{Lab}$ & 127 & .551 & .027 & {$[.50, .60]$} & & \\
\hline Field & 27 & .528 & .077 & {$[.38, .68]$} & & \\
\hline Online & 25 & .322 & .061 & {$[.20, .44]$} & & \\
\hline \multicolumn{7}{|l|}{ Manipulation } \\
\hline Real or Imagined & & & & & $3.07(1)$ & .080 \\
\hline Imagined & 110 & .561 & .034 & {$[.49, .63]$} & & \\
\hline Real & 71 & .465 & .042 & {$[.38, .55]$} & & \\
\hline Distance type & & & & & $2.07(2)$ & .355 \\
\hline Temporal & 132 & .520 & .033 & {$[.46, .58]$} & & \\
\hline Spatial & 20 & .509 & .090 & {$[.33, .69]$} & & \\
\hline Social & 23 & .638 & .077 & {$[.49, .79]$} & & \\
\hline $\begin{array}{l}\text { Dependent } \\
\text { Variable } \\
\text { Characteristics }\end{array}$ & & & & & & \\
\hline
\end{tabular}


CONSTRUAL LEVEL META-ANALYSIS

\begin{tabular}{lcccccc}
\hline Type of Outcome & & & & & $1.21(2)$ & .546 \\
Evaluations & 111 & .503 & .035 & {$[.43, .57]$} & & \\
Predictions & 30 & .583 & .067 & {$[.45, .71]$} & & \\
Behavior & 36 & .539 & .061 & {$[.42, .66]$} & & \\
\hline
\end{tabular}


Figure 1

Funnel plot of precision for the effect of psychological distance on abstraction.

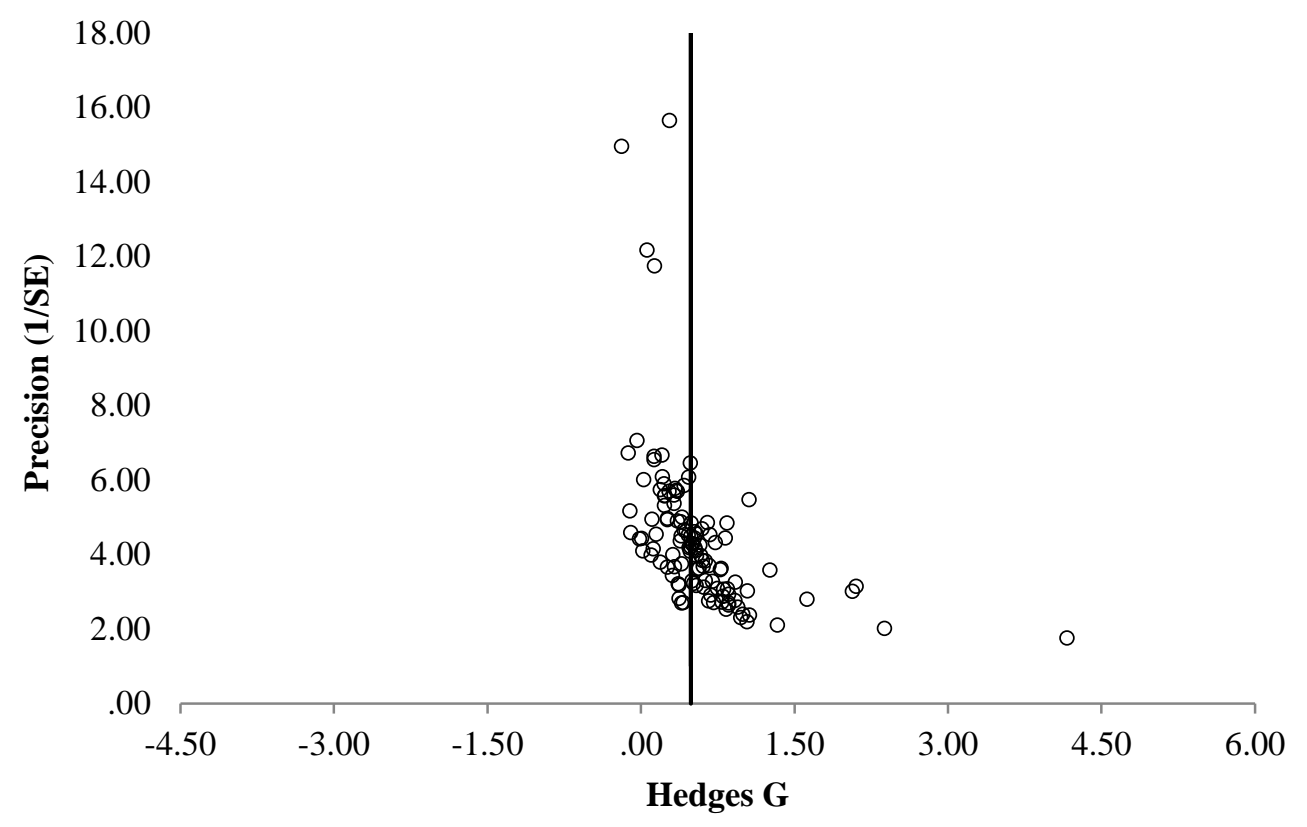


Figure 2

Illustration of the shape of the relation between future temporal distance (e.g., time until an event will occur) and construal level implied by the results of our meta-regression. The brackets provide an example of how the results in our analysis map onto the variables depicted in this graph. Imagine that the solid brackets each depict a difference of 364 days, beginning at two different time points (e.g., one day vs. 365 days for the first bracket and 183 days vs. 548 days for the second). The dotted brackets illustrate the different effect sizes that result from these one-year distance manipulations on construal level. Our results suggest the second solid bracket will produce a larger effect size than the first one (and that increasing the size of the solid bracket will also increase the effect size).

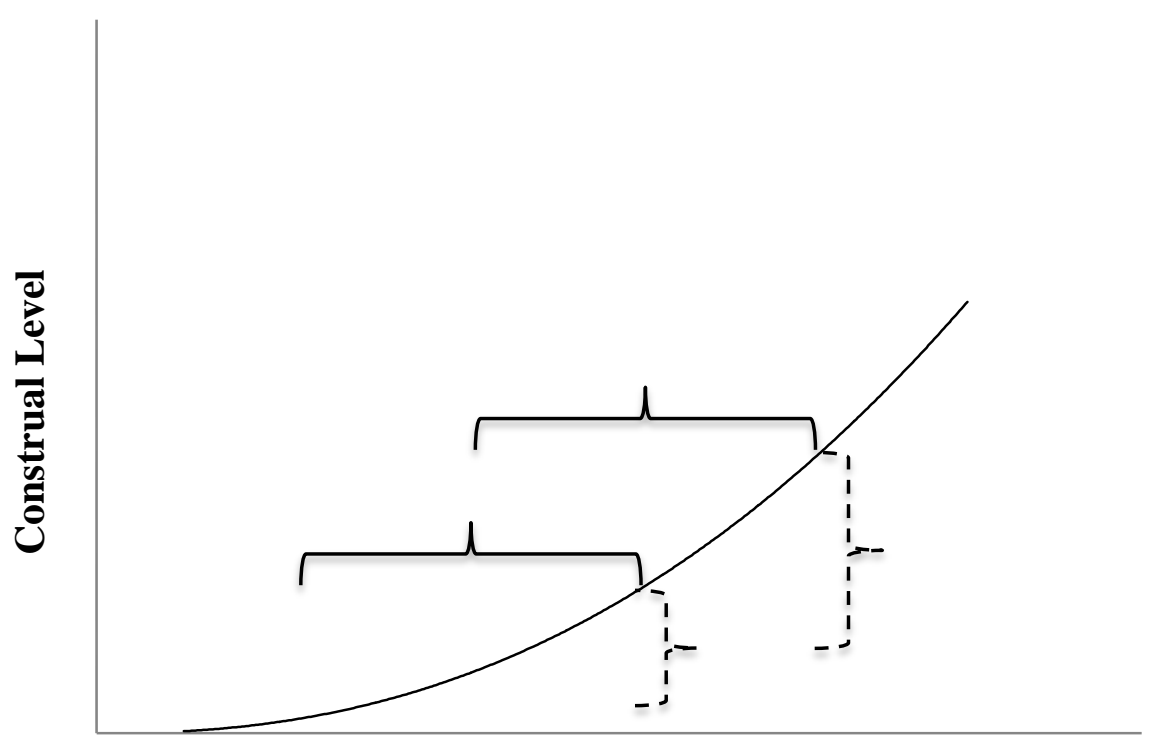

Future Temporal Distance 
Figure 3

Funnel plot of precision for the effect of psychological distance on the downstream consequences of abstraction.

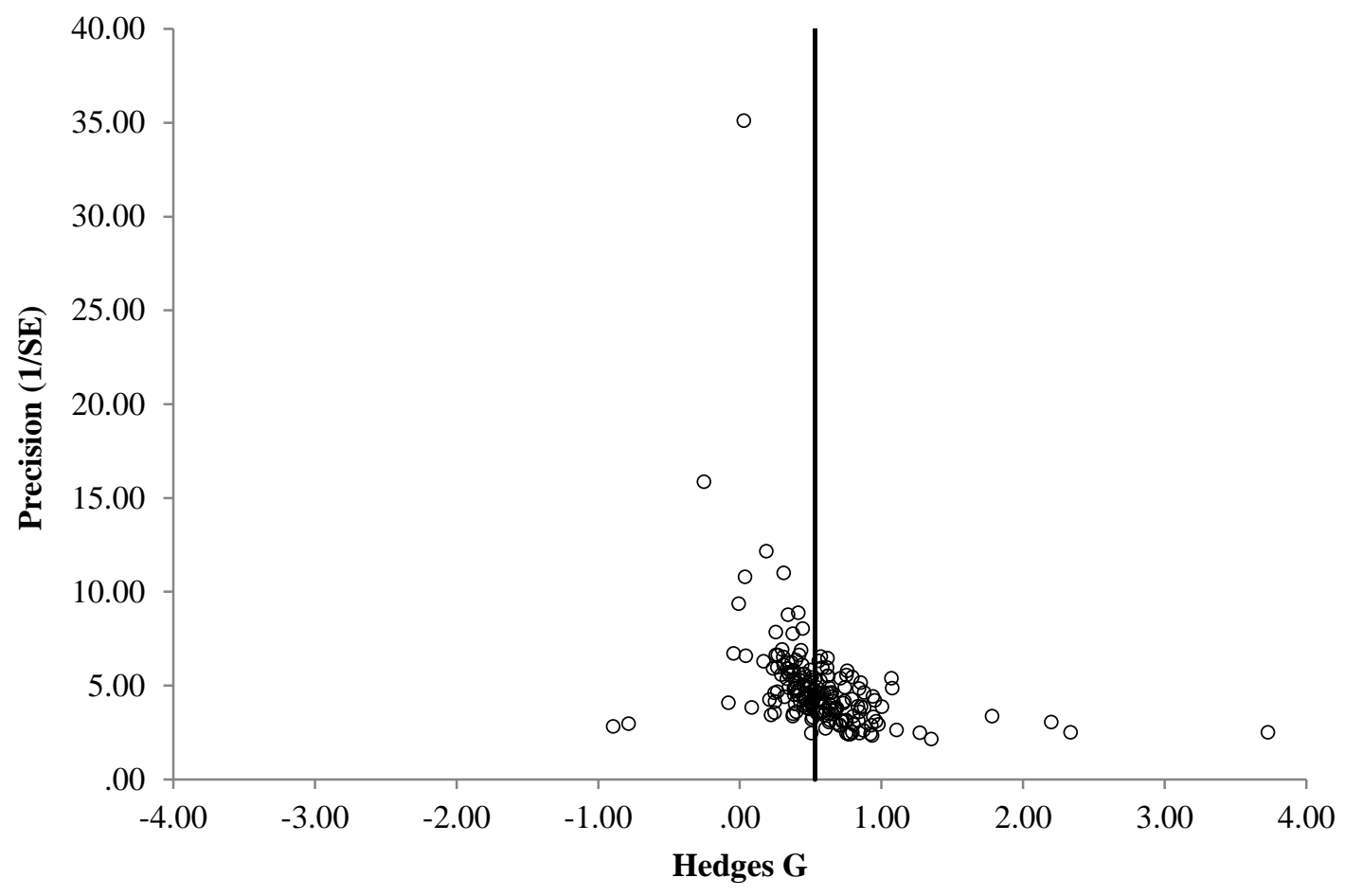


Appendix A

We conducted literature searched on PsychInfo, Pubmed, and Web of Science using the following search strings:

\section{PSYCINFO:}

KW(“construal level”) OR ("construal level theory”) OR “construal theory”)

ab(psychological distance) AND ab((abstraction) $)$

all(distance) AND all((abstraction OR "abstract thinking"))

$\mathrm{ab}$ ("construal level theory")

$\mathrm{ab}$ (“construal theory")

ab("construal") AND (“distance")

ab(“construal level”) OR (“construal level theory") OR "construal theory”) AND ("concrete")

ab(psychological distance) AND ab((“concrete"))

all(hidden figures OR embedded figures OR gestalt completion OR snowy pictures OR Analysis-Holism Scale) AND all(construal)

all(hidden figures OR embedded figures OR gestalt completion OR snowy pictures OR Analysis-Holism Scale) AND all(concrete)

all(Weschler Intelligence Task) AND all(construal OR concrete)

\section{WEB OF SCIENCE:}

Topic: "construal level theory" or "construal level"

Topic: "construal level theory" or "construal level" And abstract

Topic: psychological distance AND abstract* Spatial/social/temporal/probability/power

Topic: distance/ psychological distance AND concrete Spatial/social/temporal/probability/power 
Gestalt completion task OR BIF/Vallacher \& Wegner, 1989 OR Navon Task AND construal/abstract/concrete

Topic $=(($ hidden figures OR embedded figures OR gestalt completion OR snowy pictures OR Analysis-Holism Scale)) AND Topic=(construal)

Topic $=($ Levels of personal agency: Individual variation in action identification $)$

\section{PUBMED:}

(“construal level”) OR (“construal level theory") OR (“construal theory")

(psychological distance) AND ((abstraction)) OR ((concrete))

all((hidden figures OR embedded figures OR gestalt completion OR snowy pictures OR Analysis-Holism Scale)) AND all(construal)

Topic $=($ Levels of personal agency: Individual variation in action identification $)$ 